\title{
A nutrient-responsive hormonal circuit controls energy and water homeostasis in Drosophila
}

Takashi Koyama ${ }^{1, \dagger}$, Selim Terhzaz ${ }^{2,3, \dagger}$, Muhammad T. Naseem ${ }^{1}$, Stanislav Nagy ${ }^{1}$, Kim Rewitz ${ }^{1}$, Julian A. T. Dow ${ }^{2}$, Shireen A. Davies² and Kenneth A. Halberg ${ }^{1, *}$

${ }^{1}$ Section for Cell and Neurobiology, Department of Biology, University of Copenhagen, Universitetsparken 15, DK-2100 Copenhagen, Denmark

${ }^{2}$ Institute of Molecular, Cell and Systems Biology, College of Medical, Veterinary and Life Sciences, University of Glasgow, Glasgow G12 8QQ, UK

${ }^{3}$ MRC-University of Glasgow Centre for Virus Research, Glasgow G61 1QH, Scotland, UK.

$\dagger$, Authors contributed equally

*Author for correspondence: kahalberg@,bio.ku.dk 


\begin{abstract}
The regulation of systemic energy balance involves the coordinated activity of specialized organs, which control nutrient uptake, utilization and storage to promote metabolic homeostasis during environmental challenges. The humoral signals that drive such homeostatic programs are largely unidentified. Here we show that three pairs of central neurons in adult Drosophila respond to internal water and nutrient availability by releasing Capa-1 and -2 hormones that signal through the Capa receptor (CapaR) to exert systemic metabolic control. Loss of Capa/CapaR signaling leads to intestinal hypomotility and impaired nutrient absorption, which gradually deplete internal nutrient stores and reduce organismal lifespan. Conversely, hyperactivation of the Capa circuitry stimulates fluid and waste excretion. Furthermore, we demonstrate that Capa/CapaR regulates energy metabolism by modulating the release of the glucagon-like adipokinetic hormone, which governs lipolysis in adipose tissue to stabilize circulating energy levels. Altogether, our results uncover a novel inter-tissue program that plays a central role in coordinating post-prandial responses that are essential to maintain adult viability.
\end{abstract}




\section{INTRODUCTION}

The ability to maintain osmotic and metabolic homeostasis in response to environmental challenges is a fundamental prerequisite for animal life. Organisms must ensure a balanced equilibrium between nutrient intake, storage and expenditure, which is governed by complex interorgan communication mediated by systemic factors that coordinate the action of specialized organs ${ }^{1,2}$. Remarkably, animals that differ dramatically in life history strategy like humans and insects - engage similar homeostatic responses, such as promoting nutrient uptake during nutrient-poor states and stimulating waste excretion during nutrient-replete states. While these similarities suggest conserved underlying mechanisms, the physiological adaptations and inter-organ communication networks that drive such changes remain unresolved.

To implement the correct homeostatic program, animals must be able to sense internal nutrient abundances, and to adjust their uptake and utilization of resources accordingly. In mammals, the hypothalamus acts as a central command center for nutrient and water sensing, as it contains neuronal populations that are activated by changes in extracellular sugar concentration or osmolality and release key hormones that initiate compensatory organ activities $^{3,4}$. Similarly, in the fruit fly Drosophila melanogaster, discrete populations of neurosecretory cells function as nutrient- or osmosensors, which upon stimulation secrete neurohormones that modulate food intake, energy mobilization, gut peristalsis or renal secretion $^{5-8}$. Both mammals and flies thus regulate organ activities in response to internal state, and in many instances accomplish this regulation by similar mechanisms. However, the hormonal factors involved in coordinating systemic osmoregulation and energy homeostasis, as well as the mechanisms by which the signals are integrated by different organs are incompletely characterized. Understanding these processes is of vital importance as failure in these systems often results in fatal consequences for the organism. 
The actions of Capa signaling in insects have so far been restricted to post-embryonic stages, during which the Capa peptides are released into circulation from subsets of neurosecretory cells in the central nervous system (CNS), to activate their receptor - the Capa receptor (CapaR) - on target tissues, including the renal tubules, heart and hyperneural muscles, to control diuretic and myotropic functions ${ }^{9}$. In Drosophila, the Capa gene encodes a preprohormone that is processed to produce three different peptides, Capa-periviscerokinin1 and -2 (Capa-PVK-1 and -2) and Capa-pyrokinin-1 (Capa-PK-1) ${ }^{10}$. Strikingly, the Capa precursor is differentially processed within different subsets of neurosecretory cells, with a truncated form of Capa-PK-1 released from the subesophageal ganglion (SEG) neurons, while both Capa-PVKs and -PK are secreted from the ventroabdominal (Va) neurons ${ }^{11}$. The Capa peptides are members of the PRXamide peptide family, which is evolutionarily and functionally related to Neuromedin $\mathrm{U}(\mathrm{NmU})$ signaling in vertebrates ${ }^{12}$. In mammals, $\mathrm{NmU}$ signaling coordinates key physiological processes including visceral-muscle contractions, gastric acid secretion and insulin release as well as feeding and energy homeostasis, and is therefore an attractive therapeutic target for treating obesity ${ }^{13}$.

Here, we report that Capa-PVK signaling (hereafter just Capa) plays a key role in regulating adult osmotic and metabolic homeostasis in response to environmental conditions. Using several genetic strategies, we provide a comprehensive overview of CapaR expression, which identifies the visceral musculature and neuroendocrine cells of the corpora cardiaca (CC) - producing the glucagon analog adipokinetic hormone $(\mathrm{AKH})$ - as targets of Capa action. Loss of Capa/CapaR signaling reduces intestinal contractility, gut compartmentalization and nutrient absorption, which result in systemic metabolic defects characterized by pronounced hypoglycemia and lipodystrophy. These metabolic effects cause a gradual loss of muscle function due to dysregulated $\mathrm{Ca}^{2+}$ homeostasis in skeletal muscles, which impairs feeding behavior and cause premature death. We further show that the Capa 
Va neurons, but not the SEG neurons, secrete Capa peptides in response to nutrient availability to repress $\mathrm{AKH}$ release to restrict energy mobilization from the fat body during nutrient-replete states. We propose that the Capa Va neurons operate as post-ingestive osmoand nutrient-sensors that coordinate a CNS-gut-renal-fat body signaling module to activate two separate pathways: one to enhance intestinal and renal activities to promote energy and fluid homeostasis, and another to inhibit AKH-mediated energy mobilization to prevent hyperglycemia. Our work thus uncovers an adult-specific inter-tissue program that is essential to maintain osmotic and metabolic homeostasis in Drosophila - a program that shows remarkable functional similarity with $\mathrm{NmU}$ signaling in mammals.

\section{RESULTS}

\section{Capa/CapaR signaling is essential for adult fly survival}

To gain insight into the physiological actions of Capa/CapaR signaling, we used the binary GAL4/UAS system to silence CapaR gene expression using RNAi (Fig. S1a). Remarkably, whereas fly development progressed stereotypically (i.e. juvenile growth and developmental time are unaffected; Fig S1b-c), knockdown of CapaR expression in cells expressing this receptor using CapaR ${ }^{R N A i}$ driven by the CapaR promoter $(\text { CapaR }>)^{12}$ resulted in strong mortality shortly after adult emergence. The penetrance of phenotypic effects scaled with temperature due to the thermal flexibility of the expression system ${ }^{14}$ (Fig. 1a; median survival time was 10,3 , and 1 days at $18^{\circ} \mathrm{C}, 25^{\circ} \mathrm{C}$, and $30^{\circ} \mathrm{C}$, respectively). RNAi specificity was confirmed with two additional independent $C_{a p a R^{R N A i}}$ lines that produced similar phenotypes (Fig. S1d). However, to unambiguously demonstrate a post-developmental role of Capa signaling in sustaining adult survival, we adopted an alternative genetic strategy in which we designed a UAS-inducible tissue-specific CRISPR/Cas9 construct for CapaR (Fig. S1a), which we spatio-temporally restricted to the adult stage using the TARGET system ${ }^{15}$. 
Consistent with the phenotypes observed following CapaR>CapaR ${ }^{R N A i}$ knockdown, these data showed that adult-specific CapaR knockout $\left(\operatorname{CapaR}^{t s}>\operatorname{CapaR}^{K O}\right)$ caused complete fly mortality within 10 days following transfer to the restrictive temperature; crucially the lethality caused by CapaR deletion was almost fully rescued in flies additionally carrying a UAS-CapaR transgene immune to CRISPR-induced mutation (CapaR ${ }^{t s}>$ CapaR $^{K O}$, CapaR) (Fig. 1b). These results show that Capa/CapaR signaling is required for adult survival.

To identify the tissue-specific actions underlying this acute mortality, we selectively downregulated expression of CapaR in different tissues. Given our previous work showing that Capa peptides control renal function in Drosophila and other insects ${ }^{16}$, we initially explored the possibility that CapaR knockdown only in the Malpighian tubules (MTs) where the receptor is abundantly expressed (Fig. S2a-c) - might impact adult survival. Knocking down CapaR expression using the Uro-GAL4 driver - a GAL4 driver exclusively targeting the principal cells of MTs ${ }^{17}$ - did not cause any significant mortality (Fig. S1e), indicating that the lethality phenotype is uncoupled from Capa-dependent regulation of renal function. Indeed, expression analyses on whole animals showed a much higher reduction of CapaR mRNA levels ( $>90 \%$ knockdown) when knocked down in all CapaR-expressing cells $\left(\right.$ CapaR $>$ CapaR $^{R N A i}$ ) compared to tubule-specific knockdown (Uro $>C_{a p a R^{R N A i}} ; \square 60 \%$ knockdown), suggesting that CapaR is expressed in tissues outside the renal tubules (Fig. S1f-g). We therefore assayed the phenotypic effects of CapaR knockdown in other major tissues, including the muscles (how $>$ ), neurons (panneuronal elav $>$ or mushroom-bodyspecific $201 Y>$ ), fat body (c564> or $C g>$ ), and heart (Tinman $>$ or Hand $>$ ). These data revealed that only how $>-$ a driver that allows targeted expression in the somatic and visceral musculature (Fig. S1h-i) ${ }^{18}$ - could phenocopy the mortality observed with the CapaR $>$ driver (Fig. 1c). Importantly, this mortality phenotype was recapitulated by precise targeting of the somatic CRISPR/Cas9 construct to adult muscles (how ${ }^{t s}>$ CapaR $^{K O}$ ), which similar to global 
knockout (Fig. 1b), caused the majority of flies to die within a ten-day period. Again, this mortality rate was completely rescued to levels approaching that of controls in flies additionally expressing a transgenic CapaR receptor in the muscles resistant to the CRISPR effect $\left(\right.$ how $^{\text {ts }}>$ CapaR $^{K O}$, CapaR) (Fig. 1d). Taken together, these findings link Capa/CapaR signaling to targets outside its well-established role in regulating renal function ${ }^{12}$ and suggests that Capa peptides coordinate additional processes in the musculature that are essential to sustain adult survival.

\section{Capa signaling targets several tissues}

To identify the specific cellular targets of Capa signaling, we analyzed CapaR expression using a combination of genetic and molecular reporters. As expected, CapaR-GAL4 was found to induce strong reporter activity $(U A S-m C D 8:: G F P)$ in both the anterior and posterior MTs (Fig. S2a-c); however, we detected additional prominent and consistent GFP fluorescence in the thoracic skeletal muscles, brain as well as circular visceral muscles confined to the proventriculus, midgut and rectal regions of the intestine (Fig. 2a; Fig. S2a). The accurate reporting of endogenous CapaR localization was verified using a customsynthesized CapaR antibody ${ }^{12}$, which showed substantial overlap between GFP fluorescence and anti-CapaR immunoreactivity (Fig. 2a). These findings were further corroborated using fluorescently tagged Capa-1 peptide (Capa-1-F) in combination with a recently developed ligand-receptor binding assay ${ }^{16}$; fluorophore labelling did not affect the biological efficacy of the peptide (Fig. S2d-g). Using this approach, we observed specific and displaceable Capa-1F binding to CapaR-GAL4-driven GFP-positive visceral muscles and enteroendocrine cells (Fig. 2b-c) of the adult digestive tract, which is consistent with single-cell transcriptomic data reported by Flygut-seq ${ }^{19}$ showing CapaR expression almost exclusive in these two cell types of the gut (Fig. S2h). 
Finally, to functionally validate our receptor mapping data, we expressed an aequorinbased bioluminescent $\mathrm{Ca}^{2+}$-sensor $(\mathrm{CapaR}>$ aeq $: \because G F P)$ to detect robust changes in cytosolic $\left[\mathrm{Ca}^{2+}\right]_{\mathrm{i}}$ following Capa stimulation of acutely dissected tissues (Fig. 2d). Consistent with previous findings, Capa-1 induced a stereotypic, biphasic rise in $\left[\mathrm{Ca}^{2+}\right]$, comprising a rapid primary peak followed by a slower secondary rise in the renal tubules (Fig. S2i) ${ }^{12}$. Yet more strikingly, we also observed a prominent cytosolic calcium response in immuno-positive gut regions such as the proventriculus, midgut and hindgut/rectum, as well as in the brain (Fig. 2e-h), and to a lesser extent in the legs (Fig S2j); by contrast, we were unable to detect any $\mathrm{Ca}^{2+}$ response in the salivary glands or gonads (Fig. S2k-1). Taken together, our combined approaches provide a comprehensive overview of CapaR expression in adult Drosophila unmasking several novel target tissues of Capa neuropeptides including the skeletal and visceral muscles.

Four pairs of Capa neurons mediate brain to peripheral organ communication To determine the neurons responsible for the Capa-mediated effects on adult viability, and to enable their subsequent genetic manipulation, we characterized a new GAL4 driver line, TrpGAL4 (Trp $>$ ), that when crossed to $m C D 8:: G F P$ produced almost complete overlap in immunoreactivity with an anti-Capa precursor antibody ${ }^{10}$ in the adult CNS, hereby demonstrating unparalleled specificity and potency to the $\mathrm{Capa}^{+}$neurons (Fig. 3). Indeed, knocking down Capa expression ( $\operatorname{Tr} p>C a p a^{R N A i}$ ), or completely ablating the Capa-producing neurons via targeted expression of the proapoptotic gene $\operatorname{rpr}(\operatorname{Trp}>U A S-r p r)$ recapitulated the observed mortality phenotype of CapaR manipulations, with flies dying shortly after eclosion (Fig. S1j). Performing a detailed neuroanatomical analysis of Trp $>U A S-m C D 8:: G F P$ flies, we found consistent GFP expression in one pair of neuroendocrine cells in the SEG, whose dendritic projections innervate the AKH-producing CC cells (APCs) and extend along the 
esophagus to innervate the proventriculus (Fig. 3a-c; Fig. S3a,c). In addition, we observed reporter activity in the three pairs of Va neurons; the two anterior pairs send axons to a neurohemal plexus in the dorsal neural sheath, while the posterior-most pair join the median abdominal nerve (MAN; Fig. 3d; Fig. S3b), from which their axons project to form neurohemal release sites. Although direct innervation of the intestine by neurons exiting the CNS through the MAN or the presence of $\mathrm{Capa}^{+}$enteric neurons would offer an attractive model for Capa-mediated changes in intestinal physiology, we were unable to observe such connections. In sum, our data reveals neuronal innervation of the $\mathrm{CC}$ and proventriculus by the SEG neurons, and the systemic release of Capa peptides into circulation by the Va neurons to activate peripheral target tissues.

\section{Capa/CapaR signaling regulates gut motility as well as fluid and waste excretion}

The expression of CapaR in visceral muscles confined to portions of the adult intestine containing valves and sphincters (proventriculus, posterior midgut and rectal pad), suggests modulation of intestinal transit by Capa-dependent control of smooth muscle cell activity. Such a role is consistent with the known functions of mammalian Neuromedin U receptor 2 (NmU-R2, a functional homologue of $\mathrm{CapaR}^{12}$ ), which has been shown to promote gastrointestinal motility in mammals ${ }^{20}$. Using an established ex vivo gut-contraction assay ${ }^{16}$, we quantified the spontaneous contraction frequency of the intestine before and after Capa-1 or Capa-2 application to gut preparations. We found that both Capa peptides - at a concentration previously determined to cause maximum receptor occupation ${ }^{12}$ - stimulate gut contraction frequency approximately 2-3-fold compared to the artificial hemolymph control saline (Fig. 4a-b). Conversely, the Capa-induced activation of gut motility was abolished after CapaR knockdown $\left(\right.$ how $\left.^{t s}>\operatorname{CapaR}^{R N A i}\right)$, indicating that the increase in gut-contraction frequency following Capa-1/-2 application depends on CapaR function in intestinal muscle 
cells (Fig 4a-b). CapaR is a Gq-protein coupled receptor in which receptor occupancy results in elevation of intracellular $\mathrm{Ca}^{2+}$-levels. We therefore used the genetically encoded $\mathrm{Ca}^{2+}$ indicator UAS-GCaMP6s to directly visualize $\mathrm{Ca}^{2+}$-dynamics in CapaR-expressing circular muscle cells during Capa-1 $\left(10^{-7} \mathrm{M}\right)$ stimulation (Fig. 4c). These data show that Capa-1 application induces prominent intracellular $\mathrm{Ca}^{2+}$-transients in muscle cells supporting the notion that Capa/CapaR controls visceral muscle activity.

To explore the physiological significance of this effect, we next asked if the observed impairment of gut motility caused by reduced Capa/CapaR signaling affects intestinal transit time in vivo. In flies exposed to food supplemented with the $\mathrm{pH}$-sensitive dye Bromophenol blue (BPB), we observed a significant delay in gut transit in knockdown animals (how ${ }^{\text {ts }}>$ $\operatorname{CapaR}^{R N A i}$ ) compared to parental controls, as evidenced by the delayed appearance and significant decrease in BPB-labeled waste deposits produced over time (Fig. 4d-e). In contrast, genetic overactivation of the Capa-producing neurons using the heat-activated transient receptor potential A1 cation channel TRPA1 $(\operatorname{Tr} p>\operatorname{Tr} p A 1)$ led to a significantly shorter intestinal transit time when incubated at $30^{\circ} \mathrm{C}$ (Fig. $4 \mathrm{f}-\mathrm{g}$ ). Thus, Capa-1/-2 neurohormones communicate with the gut to modulate intestinal motility and are necessary and sufficient for the control of gut transit in adult Drosophila.

To further explore the functional significance of CapaR silencing on gut physiology in vivo, we adopted an approach that provides quantitative readouts of intestinal emptying rate and fluid content based on the semi-automated analysis of fly excreta ${ }^{21,22}$. These experiments showed that visceral muscle-specific CapaR knockdown significantly affects Drosophila defecation behavior compared to the parental controls, as these flies produced fewer, smaller, and more concentrated excreta (Fig. 4h-k). These changes in fecal output are likely caused by prolonged contact with intestinal contents associated with gut hypomotility. Consistent with this model, artificial activation of the Capa neurons $(\operatorname{Tr} p>\operatorname{Tr} p A 1)$ led to the 
production of lighter (less concentrated), larger and more abundant deposits compared to flies carrying either transgene alone, presumably due to the combined effects of intestinal hypermotility and increased diuresis (Fig. 41-o). Interestingly, limiting the time available for fluid reabsorption in the gut has a larger impact on systemic fluid balance than stimulating renal secretion, as adult-specific expression of a membrane-tethered version of the diuretic hormone DH44 (Uro >tDh44) - which allows cell-autonomous activation of its cognate receptor and thus constitutively activate tubule secretion ${ }^{23}$ - failed to fully recapitulate this diarrhea-like phenotype. Instead, the deposits produced by these flies were more numerous, but not larger or more dilute than those of controls (Fig. S4b), which is consistent with the role of the rectum in regulating the final fluid content of excreta ${ }^{24}$. Together, these results show that Capa peptides direct the actions of the CNS-gut axis by modulating intestinal contractility, which is important not only for appropriate gut transit, but also directly impacts the homeostatic regulation of water balance by affecting fluid content of excreta.

\section{$\mathrm{Capa/CapaR}$ regulates gut functions essential to maintain metabolic homeostasis}

Next, we asked if these changes in gut physiology affect the metabolic status of the fly. Indeed, in mammals gut hypomotility has been shown to compromise both intestinal acidbase balance, nutrient absorption and organismal energy status ${ }^{25}$. Consistent with these observations as well as our current findings, we observed frequent gut distension as well as loss of acidity in the copper-cell region (CCR) of midguts from Capa/CapaR deficient flies (Fig. 5a); a region functionally analogous to the mammalian stomach ${ }^{26}$. We therefore tested if these gut defects affect nutrient breakdown and absorption by the gut, by analyzing the elemental composition of deposits from flies lacking CapaR function in the visceral muscles using scanning electron microscopy coupled with wave-dispersive X-ray analysis. Interestingly, we detected significantly higher levels of carbon and nitrogen (the main 
elemental components of sugars and amino acids) in the excreta of CapaR knockdown flies compared to the control (Fig. 5b-c), suggesting a potential disruption of digestive and/or absorptive functions of the gut. To test this directly, we quantified triacidglyceride (TAG) and glucose levels in the excreta from $h o w^{t s}>\operatorname{CapaR}^{K O}$ flies, which revealed that adultspecific CapaR knockout in visceral muscles leads to significantly higher levels of residual undigested nutrients compared to controls (Fig. 5d). Importantly, these gut defects including the loss of CCR acidity - were fully rescued by co-expressing a UAS-CapaR construct $\left(\right.$ how $^{\text {ts }}>$ CapaR $^{K O}$, CapaR) (Fig. 5a,d). To further test this model, we exposed $h o w^{t s}>$ CapaR $^{K O}$ flies to more nutrient-dense foods in order to promote energy-uptake and assessed the impact of different nutrient concentrations on animal viability. These data showed that higher nutrient concentrations significantly prolonged fly survival in a dosedependent manner, whereas both control and rescue animals were unaffected (Fig. 5e). Together, our results suggest that CapaR elimination in intestinal myocytes affects the digestive and absorptive functions of the gut, which has acute effects on organismal lifespan Reduced nutrient absorption, however, could also be a compensatory reaction to excess food intake. This idea is consistent with the role of NmU signaling in regulating food consumption in rodent models ${ }^{27,28}$. To test this hypothesis, we quantified the duration of food contact as an indirect measure of food intake, in addition to directly measuring food consumption. These data revealed that CapaR knockdown flies spent significantly less time in contact with food (Fig. 5f) and that CapaR knockout animals reduced their overall dietary intake; an effect that could be reversed by overexpression of CapaR in muscles (Fig. $5 \mathrm{~g}$ ). We also observed similar effects upon targeting the four pairs of $\mathrm{Capa}^{+}$neurons by Capa knockdown $\left(\operatorname{Trp}>\mathrm{Capa}^{R N A i}\right.$; Fig. 5h), indicating that Capa/CapaR signaling play a role in regulating feeding behavior in adult flies. The combined effects of reduced energy intake and impaired intestinal absorption strongly suggest that these flies show severe metabolic defects. 
We therefore assessed the internal nutritional status of these flies, by quantifying internal carbohydrate levels (glucose and trehalose) as well as whole-body energy stores (glycogen and TAG) at distinct points in time following visceral muscle-specific CapaR elimination $\left(h o w^{t s}>\right.$ CapaR $\left.^{K O}\right)$. As expected, these data revealed a gradual decrease in internal energy stores in CapaR knockout animals, perhaps due to increasingly poor feeding and nutrient absorption, as revealed by their progressive hypoglycemic and lipodystrophic phenotypes compared to both control and rescue flies (Fig. 5i-1). Altogether, our data suggest that impairment of Capa/CapaR signaling in muscles impairs food intake and cause defects in the digestive and absorptive functions of the gut, which critically impact the metabolism and lifespan of adult flies.

Impaired Capa/CapaR signaling reduces muscle performance and adult locomotion In flies, as well as mammals, changes in systemic energy balance induces the release of hormonal factors that modulate behavioral programs and locomotor activity ${ }^{29-31}$. For example, central administration of $\mathrm{NmU}$ peptides increases gross-locomotor activity in mammals ${ }^{28}$. We therefore asked whether Capa/CapaR impairment induces similar responses in locomotor activity, by examining baseline locomotion of adult flies using the Drosophila Activity Monitor System. These data revealed that muscle-specific elimination of CapaR $\left(h o w^{t s}>\right.$ CapaR $\left.^{K O}\right)$ induced a gradual decrease in adult locomotion over the course of the 4day experimental period, as evidenced by the significant reductions in both morning and evening activity peaks relative to parental controls (Fig. 6a-b). This observation contrasts with the stereotypic increase in locomotion exhibited by energy-deprived wild-type animals, a behavior linked to food foraging ${ }^{30}$. Conversely, flies with rescued Capa signaling in muscles (how ${ }^{t s}>$ CapaR $^{K O}$, CapaR) exhibited a pronounced hyperactivity compared to control, perhaps owing to increased Capa/CapaR signaling flux in the musculature of these animals 
(Fig. 6a-b). To further evaluate the functions of Capa/CapaR signaling in muscles, we videotracked individual flies with either knockdown of CapaR in muscles $\left(\right.$ how $^{t s}>$ CapaR $\left.^{R N A i}\right)$ or in neurons $\left(\operatorname{Tr} p>C a p a^{R N A i}\right)$ and quantified the total distance traveled as well as maximal response velocity of these animals. Our data confirmed that both muscle-specific elimination of CapaR as well as Capa depletion in $\mathrm{Capa}^{+}$neurons cause significant reductions in total walking distance and maximal response velocity compared to controls (Fig. 6c-d). These data suggests that CapaR function in muscles is necessary for general motor activity in flies, and that silencing Capa signaling in muscles compromises both short-term and long-term locomotor activity.

We next explored the underlying cause of this reduced activity. Capa peptides have been shown to possess myomodulatory effects in a range of insects ${ }^{32-34}$, raising the possibility that Capa signaling may directly regulate skeletal muscle contractility in Drosophila. We therefore eliminated Capa signaling exclusively in the flight muscles by using the Act $88 F$ GAL4 driver, hereby isolating the physiological effects produced by skeletal muscles. These data showed that eliminating CapaR function in the thoracic muscles only (Act88F $>\operatorname{CapaR}^{K O}$ ) failed to: (i) reduce locomotor activity (flies with rescued Capa signaling in muscles, however, appear to be hyperactive), (ii) disrupt systemic energy homeostasis apart from reducing glycogen levels (rescue animals appear hyperglycemic); (iii) impair food intake; or (iv) induce significant mortality compared to the controls (Fig. S5a-h).

Consequently, impairing Capa/CapaR signaling exclusively in flight muscles does not recapitulate the full extent of phenotypes of global muscle knockout, suggesting that direct Capa-induced changes in skeletal muscle performance is unlikely to be the major cause to the acute mortality observed in $h o w^{t s}>\operatorname{CapaR}^{K O}$ flies.

The skeletal musculature imposes large energy demands on the organism due to its large mass and high metabolic rate. We therefore asked whether reduced systemic energy 
levels cause the observed decline in locomotor activity. First, we assessed the tissue-specific distribution of glycogen (the main energy storage form in muscles) as a functional readout of skeletal muscles' energy status using the periodic acid-Schiff (PAS) staining method ${ }^{35}$. Remarkably, PAS staining was almost completely abolished in the muscles and to a lesser extent in the fat body (but not in brain) of muscle-specific CapaR knockout flies $\left(h_{o w} w^{t s}>\right.$ CapaR $^{K O}$ ). In addition, this effect was partially rescued by co-expressing wild-type CapaR (Fig. 6e). These data confirmed that the major glycogen storing tissues (i.e. the fat body and skeletal muscles) are depleted of glycogen, which together with the generally lean phenotype of $h o w^{t s}>C_{a p a R}{ }^{K O}$ animals (Fig. 5i-1), implies that they are unable to meet the high energy demands of contracting muscles. In line with this hypothesis, we observed that the genes encoding the rate-limiting glycogenolytic enzymes Glycogen phosphorylase (GlyP) and Debranching enzyme $(A G L)$ are upregulated in CapaR knockout muscles, while the glycogenic enzymes glycogen synthase $(G l y S)$ and Branching enzyme $(A G B E)$ are significantly downregulated (Fig. 6f), indicating that the skeletal muscles are adapting metabolically by increasing glycogen mobilization in an attempt to meet autonomous energy demands.

Given that muscle performance depends critically on effective calcium cycling mechanisms, and that $\mathrm{Ca}^{2+}$-pump activity is highly sensitive to cellular energy levels ${ }^{36}$, we rationalized that energy depletion in muscle cells may compromise $\mathrm{Ca}^{2+}$ dynamics. To test this hypothesis, we quantified cytosolic $\mathrm{Ca}^{2+}$ levels during steady-state and Capa-1 stimulation in muscle-specific CapaR knockdown animals with reduced muscle performance. Strikingly, these data showed that not only does CapaR depletion abolish the Capa-induced increase in intracellular $\mathrm{Ca}^{2+}$ levels, but the unstimulated basal $\left[\mathrm{Ca}^{2+}\right]_{\mathrm{i}}$ levels were almost $50 \%$ increased relative to the control (Fig. 6g-h). This observation suggests that the transport machinery responsible for reestablishing resting $\mathrm{Ca}^{2+}$ levels is compromised. We therefore 
investigated a potential role of the Drosophila plasma membrane calcium ATPase $(P M C A)$ in muscle function - an efflux transporter crucial to maintenance of resting $\left[\mathrm{Ca}^{2+}\right]_{\mathrm{i}}$ levels and for regulating the excitation-contraction coupling process in muscles ${ }^{37}-$ and found that targeted knockdown of $P M C A$ in muscles phenocopied the dysregulated $\left[\mathrm{Ca}^{2+}\right]_{\mathrm{i}}$ levels, impaired locomotor activity and mortality phenotypes observed in how $>$ CapaR $^{K O}$ flies (Fig. S5i-1). Consistent with these observations, transcript levels of $P M C A$ were significantly reduced in CapaR knockout flies compared to control and rescue animals (Fig. 6i), suggesting that $\mathrm{Ca}^{2+}$ dysregulation in how $>\operatorname{CapaR}^{K O}$ skeletal muscles is at least partly linked with reduced PMCA activity. Overall, our data suggest that the progressive loss of muscle function observed in Capa/CapaR impaired animals is unlikely to be caused by direct Capa actions on skeletal muscles. Rather, our data point to a model in which depletion of internal energy stores and reduced $\mathrm{Ca}^{2+}$-pump activity disrupts $\mathrm{Ca}^{2+}$-dynamics in muscles, creating a muscular dystrophy-like phenotype analogous to that observed in human myopathies ${ }^{38}$.

\section{Nutrients and water induce release of Capa peptides}

Our data imply that Capa/CapaR signaling plays a central role in coordinating postprandial homeostasis, such as by increasing gut peristalsis, facilitating nutrient absorption and increasing renal secretion. We therefore tested if the Capa-expressing neurons are sensitive to internal signals related to water and nutrient availability. This hypothesis is consistent with the observation that Capa peptides accumulate in Va neurons during desiccation stress, but are subsequently released during recovery ${ }^{39}$. We thus exposed flies to either desiccation or starvation for 24 hours followed by transfer to media with different water and nutrient compositions and applied complementary approaches to measure neurosecretory activity (Fig. 7a). First, we quantified Capa transcript levels as well as intracellular Capa prohormone levels in $\mathrm{Capa}^{+}$neurons. Secondly, we used an in vivo calcium reporter (CaLexA) that 
translates sustained neural activity into nuclear import of the chimeric transcription factor LexA::VP16::NFAT and thus to subsequent LexAop-GFP expression ${ }^{40}$. Consistent with previous findings, these data showed that Capa mRNA levels and immunoreactivity were significantly increased in Va neurons, but not SEG neurons, of desiccated flies compared to control, yet returned to steady-state levels or lower 4 hours after refeeding or rehydration (Fig. 7b-c; Fig. S6). As expected, we observed inverse changes in activity in flies carrying Trp-GAL4 and UAS-mLexA::VP16::NFAT in Capa-expressing neurons (Fig. 7d). These data suggest that the Va neurons are inactive during periods of water and/or nutrient restriction but are induced to release Capa neuropeptides at high rates following both refeeding and rehydration. Interestingly, however, long-term hydration of rehydrated flies led to gradual accumulation of Capa peptides, and a concomitant reduction in GFP intensity over the ensuing 24 hours (Fig. 7c-d), indicating that water ingestion alone is insufficient to maintain stimulation of Capa-Va neuronal activity in hydrated flies.

Since starvation affects Capa release, we tested whether the $\mathrm{Capa}^{+}$neurons were sensitive to nutritional cues. In flies exposed to nutrient-deprivation for 24 hours, we observed a significant increase in Capa mRNA levels and Capa immunoreactivity and a concomitant reduction in GFP reporter expression in the Va neurosecretory cells, with both Capa and GFP intensities returning to steady-state levels following refeeding on standard medium (Fig. 7b-d). These data demonstrate that the Capa ${ }^{+}$Va neurons, and again not the SEG neurons (Fig. S6), are responsive to signals related to internal nutrient availability. To further identify the dietary factors that regulate Capa release, we transferred starved flies to media that contained either sucrose and yeast as exclusive sources of carbohydrates or amino acids, respectively. Interestingly, refeeding sugar, but not amino acids, reverted anti-Capa and GFP fluorescence levels similar to the ones observed in fully fed conditions (Fig. 7c-d), suggesting that the Va neurons are specifically activated in response to dietary sugars or their 
metabolites. Together, our data show that the different populations of $\mathrm{Capa}^{+}$neurons are differentially activated: only the three pairs of $\mathrm{Capa}^{+} \mathrm{Va}$ neurons relay information about the internal availability of water and sugar levels, because only these neurons release Capa peptides into the circulation in response to environmental conditions known to affect the internal abundance of these nutrients.

Capa-mediated regulation of AKH secretion controls systemic metabolic homeostasis The finding that Capa neurons respond directly to internal sugar levels implies that Capa peptides relay nutritional information to exert metabolic control. This prompted us to explore a potential interaction between Capa signaling and hormonal systems controlling systemic energy balance. As in mammals, the Drosophila insulin-like peptides (DILPs) and AKH (a glucagon analogue) act as the key counter-regulatory hormones in controlling carbohydrate metabolism ${ }^{8,30,41}$. Although we were unable to detect GFP expression in the adult insulinproducing cells (Fig. 2a), we surprisingly found reporter-gene expression (CapaR $>G F P)$ in the $\mathrm{AKH}$-producing neurosecretory cells in the $\mathrm{CC}$; the sole site of $\mathrm{AKH}$ synthesis and release $^{30}$. Furthermore, we observed specific and displaceable Capa-1-F binding overlapping with APC-targeted GFP expression $(A k h>G F P)$ (Fig. 8a-b). These data suggest that Capa peptides regulate energy metabolism by directly modulating the AKH signaling pathway. Because the primary function of $\mathrm{AKH}$ is to induce lipid catabolism, which affects organismal resistance to starvation, we measured survival under starvation in animals with reduced CapaR expression in the APCs to test whether Capa regulates AKH secretion. Flies with adult-specific knockout of CapaR in the APCs $\left(\right.$ Akh $\left.^{t s}>\operatorname{CapaR}^{K O}\right)$ showed a marked hypersensitivity to nutrient deprivation compared to age-matched controls $\left(A k h^{t s} /+\right)$, an effect previously associated with increased $\mathrm{AKH}$ release ${ }^{30}$; we furthermore observed that this effect was fully rescued in flies additionally carrying a UAS-CapaR transgene (Fig. 8c). These data 
show that Capa mediates adult-specific nutritional regulation of $\mathrm{AKH}$ signaling, which is necessary to sustain organismal survival during nutrient deprivation.

To further probe the interactions between AKH and Capa signaling, we compared $A k h$ transcript and intracellular AKH protein levels upon APC-specific CapaR knockout in flies exposed to either ad libitum feeding or caloric deprivation. Neither starvation nor CapaR manipulation altered $A k h$ expression (Fig. 8d). However, starvation induced a marked decrease in intracellular AKH levels in the APCs in all genotypes, consistent with the known induction of AKH release by starvation ${ }^{30}$ (Fig. 8e). Moreover, in both fed and starved conditions we also observed that $\operatorname{CapaR}$ knockout $\left(\mathrm{Akh}^{t s}>\mathrm{CapaR}^{\mathrm{KO}}\right)$ consistently lowered intracellular AKH protein levels relative to control, which was reverted to baseline levels in rescue animals $\left(A k h^{t s}>C_{C a p a R}{ }^{K O}\right.$, CapaR $)($ Fig. 8e). These data demonstrate that CapaR activation in the APCs act to repress release of AKH into circulation in a nutrient-dependent manner, and that the Capa neuroendocrine pathway thus exerts metabolic control by modulating AKH activity. Such a function is furthermore consistent with the sugar-induced release of Capa from the Va neurons given that $\mathrm{AKH}$ release should be inhibited during sugar-replete states, in part via Capa/CapaR, in order to prevent lipolysis.

Given that the main downstream action of $\mathrm{AKH}$ is to regulate lipolysis in adipose tissue, we investigated the metabolic status of CapaR-deficient animals by assessing their lipid reserves in the adult fat body. Using the lipophilic dye Nile Red to direct visualize lipid contents $^{42}$, we observed a significant but rescuable reduction in fat body lipid-droplet size in CapaR-knockout animals under both fed and starved conditions; starvation also induced a partially rescuable decrease in lipid-droplet number in $A k h^{t s}>C_{a p a R}{ }^{K O}$ animals (Fig. 8f-h). Consistent with these observations, we detected a significant reduction in organismal energy reserves in both fed and starved animals compared to control, as indicated by decreased whole-body TAG levels in APC-specific CapaR knockout animals (Fig. 8i,k). In addition, fed 
flies lacking CapaR in the APCs displayed a pronounced hyperglycemia relative to control and rescue animals, consistent with hyperactivation of AKH signaling in these flies. In contrast, starved flies exhibited a decrease in glycemic levels, likely due to an accelerated depletion of organismal energy reserves (Fig. 8j,1). Altogether, our results identifies an intertissue program involving $\mathrm{Capa}^{+}$Va neurons, which sense internal sugar and water availability and signal to neurosecretory cells in the $\mathrm{CC}$ to control $\mathrm{AKH}-$ mediated catabolic processes in adipose tissue, adding another layer of regulation to the complex control of energy homeostasis in the adult fly.

\section{DISCUSSION}

Between what goes in and what goes out: Capa signaling directs post-ingestive intestinal physiology and excretory behavior

Our work has identified a system in which Capa peptides released by six Va neuroendocrine cells of the ventral nerve cord activate their receptor CapaR localized to visceral muscles to control intestinal motility and food transit. Activating the Capa circuit increases intestinal transit causing flies to excrete large amounts of dilute deposits, whereas inactivation of CapaR in gut muscle cells results in reduced gut motility and excretion. These physiological effects are linked with the strategic localization of CapaR expression to visceral muscles in distinct regions of the gut that contain valves and sphincters ${ }^{22}$, implying that Capa peptides are part of a digestive program by which the transit of intestinal contents is sensed and modulated. Consistent with this idea, genetic inhibition of gut peristalsis and intestinal passage appear to increase the amount of fluid absorbed by the gut, which leads to a concentration of waste products and causes a constipation-like phenotype analogues to the effects observed in humans suffering from gastrointestinal motility disorders ${ }^{25}$. This manipulation also frequently associates with intestinal distention, which incidentally helps 
explain the reduced food intake by these flies, given that enteric neurons convey stretchdependent feeding-inhibitory signals ${ }^{43,44}$. Our work and previous studies ${ }^{12,45}$ are therefore consistent with a model in which the synchronized control of renal secretion and gut motility by the Capa circuit helps synergize the homeostatic control of water balance and waste excretion, which shows striking functional homology to the actions of $\mathrm{NmU}$ signaling in humans ${ }^{13}$. Intriguingly, the coupled control of renal and gut functions appears to have been co-opted during evolution since other hormones that exert systemic control of diuresis - such as kinin, Diuretic Hormone 31 (DH31) and Diuretic Hormone 44 (DH44) - have also been reported to modulate feeding behavior and intestinal contractions ${ }^{6,44,46,47}$. The convergence of multiple neuropeptide signaling pathways on organ functions critical to controlling postingestive physiology, suggests a fundamental need for coupling food intake with fluid and waste excretion. How these endocrine networks interact to regulate these vital processes remains to be examined.

In addition to compromising intestinal fluid balance and excretion, we also found that silencing Capa-mediated visceral contractions disrupt the integrity of the midgut acidic zone. The acidic zone consists of acid-secreting copper cells that are functionally related to the gastric parietal cells of the mammalian stomach, and this region is necessary to facilitate the digestion and absorption of macronutrients and metals ${ }^{26,48,49}$. Although we cannot exclude that the loss of acidity is due to reduced copper-cell acid secretion, our data suggests that CapaR expressing visceral muscles at the anterior and posterior junctions of this region serve a sphincter-like role that is necessary to maintain gut compartmentalization and optimal $\mathrm{pH}$ of the CCR; a role strikingly similar to that played by the esophageal and pyloric sphincters in humans. This idea is consistent with the morphological constrictions of the gut tube at either end of the CCR and is supported by a recent study demonstrating that ablation of enteroendocrine derived DH31, which controls food passage into the acidic zone in 
Drosophila larvae, leads to inappropriate mixing of acidified and non-acidified gut contents $^{48,50}$. Our data thus point to a mechanism in which nutrient-dependent Capa release helps maintain functional gut compartmentalization in order to promote and optimize nutrient digestion and uptake following food consumption.

Capa-mediated control of systemic metabolism affects locomotor activity and survival Silencing of Capa/CapaR signaling in muscles induces a complex metabolic phenotype characterized by lipodystrophy and hypoglycemia accompanied by hypophagia, locomotor deficits, and reduced organismal lifespan. How can we reconcile these phenotypes? Several lines of evidence indicate that impaired nutrient uptake is a conserved mechanism reducing organismal longevity ${ }^{51,52}$, raising the possibility that these phenotypes arise as a consequence of malnutrition and poor intestinal absorption. This idea is consistent with the gradual decrease in whole-body energy stores following conditional CapaR knockout in visceral muscles and is further supported by the observation that survival can be enhanced by exposing these flies to higher nutrient concentrations. Concordantly, our data show that the loss of internal metabolic stores correlates with the progressive decline in physical activity. These results strongly suggest that the age-dependent decline in locomotor activity derives from impaired energy homeostasis affecting the musculature. Intriguingly, locomotor deficits are known to impair foraging behavior and food intake ${ }^{53}$, suggesting that the reduced muscle performance observed in Capa/CapaR deficient animals may contribute to the hypophagia exhibited by these animals, causing further malnutrition and ultimately death. Altogether, our data support a model in which nutrient absorption is one of the key aspects of Capa-mediated control of adult metabolic homeostasis - a function that is essential to sustain physical activity, feeding and survival, 
Coupled sensing of internal sugar and water abundance for maintaining homeostasis

Our study suggests that the $\mathrm{Capa}^{+}$Va neurons, but not the SEG neurons, secrete Capa peptides in the presence of high internal water and sugar levels, while desiccation and caloric deprivation reduce release and thus results in Capa hormone accumulation. These data connect homeostatic variations in internal water and sugar availability to Capa signaling, and remarkably show that the Capa Va and SEG neurons are activated by different physiological signals. Yet, how might this type of regulation be adaptive? One possibility is that food and water consumption change the internal metabolic status of animals (in a manner dependent on food composition), requiring appropriate activation of specialized organs to restore homeostasis. For example, feeding in Drosophila is associated with fluid uptake, which needs to be balanced by renal excretion to avoid acute effects; prolonged inactivation of diuresis can cause severe fluid retention, resulting in cuticle rupture and death ${ }^{22}$. Conversely, nutrient and water deprivation induce osmotic perturbations requiring compensatory actions to reduce organismal water loss; inhibiting tubule and intestinal activities promotes fluid retention and increase survival under desiccating conditions ${ }^{45,54}$. Our study thus provides evidence of a mechanism by which homeostatic deviations in internal osmolality may be reported by the $\mathrm{Capa}^{+}$Va neurons, which provide systemic feedback regulation on intestinal and renal activities to restore fluid and ion balance.

Our finding that $\mathrm{Capa}^{+} \mathrm{Va}$ neurons are potently activated by high hemolymph sugar levels - a conserved signal of energy surfeit - and that Capa modulates AKH release and thus govern lipolytic activity in the fat body, surprisingly implicate Capa action in glycemic control. Our results consistently show that impaired Capa signaling in the APCs due to CapaR elimination induces a marked hypersensitivity to starvation; a phenotype that is also associated with a significant increase in AKH secretion, reduced fat body energy reserves, and hyperglycemia (Fig. 8c-j). Whereas the impact of insulin regulation on carbohydrate 
metabolism is well studied in both humans and flies, less is known about AKH/glucagon. Our study reveal a neuroendocrine mechanism in which Capa peptides released from the CNS exert metabolic control by providing feedback regulation on AKH-mediated lipolysis in the fat body to stabilize circulating sugar levels. Intriguingly, similar suppression of a metabolic hormone was recently reported for $\mathrm{NmU}$ signaling in mammals ${ }^{55}$, implying that vertebrates and invertebrates may share evolutionarily conserved mechanisms for regulating sugar metabolism.

A key requirement of homeostatic regulation is the ability to sense deviations in internal abundances and to initiate compensatory actions to restore balance. Although we do not know whether the $\mathrm{Capa}^{+}$Va neurons are directly sugar- and osmosensitive, or alternatively if such information is encoded by other neurons, it is surprising that cues related to both sugar and water availability converge on the same group of neurons. Strikingly, a recent study found that expression of AKHR, and the TRPV channel, Nanchung, by the same group of interoceptive neurons underlie their ability to direct monitor internal signals of nutrient and water availability ${ }^{7}$. Similarly, sugar and water reward have also been found to be processed by several other subsets of neurons in the brain, in a manner independent of gustatory sensory activation $6,8,56,57$. These data imply that several internal nutrient sensors in the Drosophila brain communicate homeostatic needs for sugar and water independently, and that Capa signaling may interact with other signaling networks to couple consumption with the regulation of absorptive and excretory organs to restore balance. Indeed, it is likely that in the face of complex environmental challenges, multiple mechanisms converge to ensure a robust organismal response to diverse stressful conditions to sustain animal survival.

In sum, our work has uncovered a key role for Capa signaling in coordinating organspecific post-prandial responses that are essential to sustaining adult fly survival. This interorgan signaling system consists of a CNS/gut/renal/fat body signaling module, which 
operates via a discrete population of sugar- and osmosensitive $\mathrm{Capa}^{+}$neurons that release Capa peptides into circulation to control gut peristalsis, nutrient uptake, renal secretion and fat body lipolysis, to ultimately promote internal osmotic and metabolic homeostasis following sugar and water ingestion (Fig. $8 \mathrm{~m}$ ). Overall, the striking functional conservation observed between insect Capa and mammalian NmU signaling further emphasizes the unique power of the Drosophila model system as a tool that may provide valuable insights into the complex regulation of metabolic and energy homeostasis in humans.

\section{MATERIALS AND METHODS}

Fly stocks. UAS- $\operatorname{CapaR}^{R N A i}$ (2727), UAS-mCD8::GFP (5137), UAS-mLexA-VP16-NFAT (66542), UAS-GCaMP6s (42749), elav-GAL4 (458), Cg-GAL4 (7011), 201 Y-GAL4 (4440), c564-GAL4 (6982), how(24B)-GAL4 (1767), Akh-GAL4 (25684), UAS-Tubulin-Gal80ts (7019), Act88F-GAL4 (38461) and Trp-GAL4 (49296) flies were obtained from Bloomington Drosophila Stock Center (Indiana University, Bloomington, USA). Moreover, UAS$\operatorname{CapaR}^{R N A i}(105556)$ and $P M C A^{R N A i}$ (101743) transgenic fly lines were obtained from the VDRC RNAi stocks (Vienna Drosophila Resource Center, Austria). Tinman-GAL4 was kind gift from Dr. Manfred Frasch (University of Erlangen-Nuremberg, Germany) and HandGAL4 was kind gift from Dr. Achim Paululat (University of Osnabrück, Germany). The CapaR-GAL4, CapaR-GAL4;UAS-GFP::aequorin, how(24B)-GAL4;UAS-GFP::aequorin, and Uro-GAL4 drivers were previously generated in-house. In addition, a viable doubly homozygous CapaR RNAi (II, III) fly line was generated using standard genetic techniques. All fly lines were maintained on a standard Drosophila cornmeal medium at $25^{\circ} \mathrm{C}, 50-60 \%$ humidity under a 12-hour:12-hour light-dark photoperiod. Standard fly crosses were performed at $25^{\circ} \mathrm{C}$. Flies carrying the TubulinGal80 $0^{t s}$ transgene encoding a temperaturesensitive transcriptional repressor were raised at $18^{\circ} \mathrm{C}$ and subsequently sorted for males and 
females upon eclosion. After sorting, flies were kept for another 24 hours at $18^{\circ} \mathrm{C}$ before being switched to $30^{\circ} \mathrm{C}$ for maturation. Only male flies were used for experimentation. $w^{1118}$ was used as wild-type control.

Drosophila transgenesis. The ORF of CapaR (CG14575) was amplified from whole $w^{1118}$ fly cDNA as template and cloned into pUAST attB vector using NotI and KpnI whose recognition sites are included on the primers (Table S1), then integrated on the second chromosome by site-directed insertion using the phiC31 integrase and an attP landing site carrying recipient line, $y[1] w[1118] ; P B a c\left\{y[+]-a t t P-9 A^{58}\right.$ VK00018\} (Bloomington Drosophila Stock Center \#9736). The tissue-specific CapaR CRISPR ${ }^{\text {KO }}$ construct was cloned into pCFD6 vector (www.crisprflydesign.org, Addgene \#73915) according to the website's instruction. In this vector, we cloned four independent gRNA constructs designed to target non-coding regions (introns and 3'UTR; see primers listed in Table S1) of the CapaR genomic DNA sequence. After sequencing, the construct was inserted on the third chromosome in an attP2 landing site carrying recipient line, $y, w, P(y[+] . n o s-i n t . N L S)$; $P($ CaryP)attP2 (gift from Dr. Diogo Manoel, Instituto Gulbenkian de Ciência, Portugal). Germline transformation was carried out in-house.

Survival assay. Flies from each genetic background were collected within 24 hours after eclosion and maintained on standard Drosophila cornmeal medium (app. 30 flies per vial) at either 18,25 or $30^{\circ} \mathrm{C}$ at $50-60 \%$ humidity with a 12 -hour:12-hour light-dark photoperiod. In all conditions, survivors were transferred into new vials every three days. Each vial was then observed for dead flies (no movement after gently tapping the vial) every 24 hours for 10 days. Data was expressed as per cent survival over time with 213-793 flies counted for each genotype and condition. 
Quantitative real-time PCR analysis. Total RNA was extracted from whole animals or dissected tissues using the RNeasy Mini Kit (QIAGEN) with DNase treatment to remove contaminating genomic DNA. RNA was quantified using a NanoDrop spectrophotometer (Thermo Scientific) and reverse transcribed into cDNA using iScript Reverse Transcription Supermix (Bio-Rad). Quantitative real-time PCR (qPCR) was performed using the QuantiTect SYBR Green PCR Kit (Qiagen) and the Mx3005P qPCR System (Agilent Technologies) with transcript levels normalized to $R p L 32$ or $R p L 3$ reference genes and expressed as fold change compared to controls \pm s.e.m $(n=5)$. The primers used are listed in Table S2.

Whole fly paraffin-embedded sections and immunostaining. Paraffin sections were made according to a modified protocol previously described ${ }^{59}$. In brief, CapaR $>$ GFP flies were fixed in 4\% (wt/vol) paraformaldehyde (PFA) for 30 minutes, and then dehydrated through a graded series of ethanol and xylene, before being embedded in paraffin. Next, $\sim 8 \mu \mathrm{m}$ thick paraffin sections were cut on a Leica RM 2235 microtome (Leica Biosystems, Nussloch, Germany), and the sections were transferred to individual glass microscope slides for subsequent use. For immunostainings, the sections were deparaffinized in Histo-Clear (National Diagnostics, USA) for 15 minutes, rehydrated through a graded series of alcohol (99\%, 90\% and 70\% for 10 minutes each step), and rinsed in PBS. The sections were then incubated in blocking buffer (PBS with $0.1 \%$ Triton X-100 and $2 \%$ normal goat serum), containing polyclonal Alexa Fluor 488-conjugated rabbit anti-GFP (1:200; ThermoFisher, \#A21311) and DAPI (4',6-diamidino-2-phenylindole, $1 \mu \mathrm{g} \mathrm{ml}^{-1}$; ThermoFisher, \#D1306) overnight at $4^{\circ} \mathrm{C}$. Finally, the sections were washed repeatedly in PBS and mounted in Vectashield (Vector Laboratories Inc, CA, USA). Image acquisition was performed on an 
inverted Zeiss LSM 880 confocal laser-scanning microscope (Zeiss, Oberkochen, Germany) and processed with CorelDraw X8.

Immunocytochemistry. Immunocytochemistry was performed as previously described ${ }^{60}$. In brief, Drosophila tissues were dissected in Schneider's insect medium (Invitrogen, CA, USA), fixed in 4\% (wt/vol) paraformaldehyde in PBS for $30 \mathrm{~min}$ at room temperature and washed twice for 1 hour in blocking buffer. Tissues were then incubated in primary antibodies in blocking buffer overnight, followed by overnight incubation in secondary antibodies in blocking buffer at $4^{\circ} \mathrm{C}$. Counter staining with DAPI and/or Rhodamine-coupled phalloidin (1:100; ThermoFisher, \#R415) was performed where appropriate. The primary antibodies used were Alexa 488 conjugated goat anti-GFP (1:500), rabbit anti-CapaR ${ }^{12}$ (1:500), rabbit anti-Capa precursor peptide ${ }^{10}$ (1:500) and rabbit anti-AKH (raised against the secreted portion of the peptide ${ }^{30}$ ), a generous gift from Dr. Jae Park, University of Tennessee, US (1:500). The primary antibodies were visualized with goat anti-rabbit Alexa Fluor 488, 555 or 594 (1:500; ThermoFischer \#A32731, \#A21429 or \#R37117). For staining of intracellular lipid droplets in the fat body, adult adipose tissue was fixed as described above and the lipophilic dye Nile red $(2.5 \mu \mathrm{g} / \mathrm{ml})$ was applied for 30 minutes after which tissues were rinsed with PBS. All samples were mounted on poly-L-lysine coated $35 \mathrm{~mm}$ glass bottom dishes (MatTek Corporation, MA, USA) in Vectashield and imaged as previously described. Retained intracellular Capa prohormone and AKH peptide levels as well as lipid droplet size and number were calculated using the FIJI software package.

Peptide synthesis and ex vivo receptor-binding assay. Drosophila Capa-1 and Capa-2 (both with and without an N-terminal cysteine) were synthesized by Cambridge Peptides (Birmingham, UK), and subsequently coupled to high quantum yield fluorophores via a 
cysteine-linker to make fluorescent TMR-C5-maleimide-GANMGLYAFPRVamide (Capa-1-

F), and Alexa488-C5-maleimide-ASGLVAFPRVamide (Capa-2-F). Fluorescent Capa

peptides were applied to tissues in an ex vivo receptor-binding assay as previously

described ${ }^{16}$. In brief, tissues of interest were dissected from cold anesthetized animals and mounted on poly-L-lysine coated glass bottom dishes before being setup in a matched-pair protocol. One batch was incubated in 1:1 (vol/vol) mix of Drosophila saline and Schneider's medium (artificial hemolymph; AHL) containing DAPI, while to the other batch was additionally added $10^{-7} \mathrm{M}$ of Capa-1-F. The batch without the labeled peptide was used to adjust baseline filter and exposure settings to minimize background during image acquisition on a Zeiss LSM 880 confocal microscope. Specificity of binding was additionally verified by competitive displacement of the labeled ligand with unlabeled peptide at a concentration of $10^{-5} \mathrm{M}$.

Real-time measurements of intracellular calcium release. Cytosolic calcium measurements were performed according that previously described ${ }^{61}$. Tissues were dissected from flies expressing UAS-GFP::aequorin driven by CapaR-GAL4 or how-GAL4. For each sample, 20-30 live intact adult tubules, proventriculus, midgut, hindgut, brain, gonads, salivary gland, legs or thoraxes were transferred to $5 \mathrm{ml}$ Röhren tubes (Sarstedt AG \& Co., Nümbrecht, Germany) in $175 \mu$ l Schneider's medium and subsequently incubated in the dark with $2 \mu \mathrm{l}$ coelenterazine in ethanol (final concentration of $2.5 \mu \mathrm{M}$ ) for 3 hours to reconstitute active aequorin. Real-time luminescence was measured on a Berthold Lumat LB 9507 luminometer (Berthold technologies, Bad Wildbad, Germany). A stable baseline was established prior to both mock injection and subsequent injection with Capa-1 peptide (final concentration $10^{-7} \mathrm{M}$ ), and the luminescence was measured in the ensuing period. After each experiment, undischarged aequorin was measured by permeabilizing the cells with $300 \mu 1$ 
lysis buffer $\left(1 \%\right.$ Triton $\mathrm{X}-100$ and $\left.0.1 \mathrm{M} \mathrm{CaCl}_{2}\right)$. Real-time calcium concentrations $\left(\left[\mathrm{Ca}^{2+}\right]_{\mathrm{i}}\right)$ throughout the experiments were then back-calculated with an in-house PERL routine.

Gut motility assay. Guts from control and CapaR knockdown flies were dissected in artificial hemolymph (AHL) with minimal disruption of attached tissues and without removing the head. Individual exposed guts were next pinned onto a Sylgard-lined petri dish, with fine tungsten pins through the proboscis and a small piece of cuticle attached to the end of each gut and bathed in $100 \mu \mathrm{l}$ of AHL. Following a 5-minutes acclimation period, each gut was then recorded with a Leica IC80 HD camera mounted on a Leica M50 stereomicroscope for 3-minutes, before the solution was exchanged with $100 \mu 1$ of AHL and recorded for another 3-minutes. This was done to correct for potential artefacts on gut contraction frequency associated with the exchange of solutions. Next, the AHL solution was replaced with $100 \mu \mathrm{l}$ of $10^{-7} \mathrm{M}$ of either Capa-1 or Capa-2 peptide in AHL, and tissues recorded for an additional 2 x 3-minutes. Contractions of the entire gut were visually counted post image acquisition, and the number of contractions was normalized to the number of contractions observed in the initial AHL solution with $n=6-8$ guts counted for each genotype.

Calcium imaging. To measure muscle activity upon Capa peptide stimulation, guts from CapaR $>U A S-G C a M P 6$ s flies were dissected in AHL and immobilized by mounting in polyL-lysine coated dishes. Each gut was recorded for 500 frames in total (512 x 512 pixels; one frame per 5 seconds); the initial 100 frames were recorded prior to Capa peptide stimulation, the next 200 frames were recorded during stimulation with $10^{-7} \mathrm{M}$ Capa-1, and the final 200 frames were recorded during wash-out. 
Quantitative analysis of defecation behavior. Analysis of defecation behavior was performed according to a modified protocol ${ }^{22}$. Standard Drosophila food was prepared and allowed to cool to roughly $65^{\circ} \mathrm{C}$ before it was supplemented with $0.5 \%$ (wt/vol) Bromophenol blue (BPB) sodium salt (B5525, Sigma). Both gut transit and excreta quantification experiments were performed on batches of 10 flies, which were placed in standard fly vials or 50-mm Petri dishes containing BPB-dyed food. For gut transit measurements, the flies were transferred to fresh vials every hour and the number of excreta manually scored. For Petri dishes, digital images of Petri lids were obtained using an photo scanner (Konica C454e), which were processed in ImageJ before being analyzed (including depsit number, size, and dye intensity) using the TURD software package ${ }^{21}$. Dissections of dye-containing intestines were performed in AHL, leaving the head and posterior cuticle intact to prevent leakage of gut contents.

Scanning electron microscopy and quantitative elemental microanalysis. Deposits were collected and transferred to individual aluminium stubs. Samples were then examined in a Zeiss Sigma variable pressure analytical scanning electron microscope (Carl Zeiss, Oberkochen, Germany) in combination with AZtecEnergy microanalysis software (Oxford instruments, Oxford, UK). Element microanalysis and acquisition of composition maps was carried out using high accelerating voltages $(\geq 20 \mathrm{kV})$ in combination with electron backscatter (BSE) and both energy-dispersive X-ray spectroscopy (EDS) and wavelengthdispersive X-ray spectroscopy (WDS), providing both structural and quantitative measures for excretion composition at high resolution.

Feeding assays. Groups of flies were starved for 4 hours, and then transferred to normal food containing $0.5 \%$ BPB for another 4 hours, followed by freezing the flies at $-80{ }^{\circ} \mathrm{C}$. Groups of 
five flies were transferred to $2 \mathrm{~mL}$ tubes and were homogenized in $100 \mu \mathrm{PBS}+0.1 \%$ Triton X-100 using a TissueLyser LT (Qiagen). The samples were then spun down at 12,000 rpm and the supernatant transferred to fresh vials and the absorbance of each sample at $603 \mathrm{~nm}$ was measured using a NanoDrop. The resulting absorbance values were then subtracted the mean background value obtained from unfed flies $(n=10)$, and the data were normalized to control. A total of $n=22-27$ samples were measured for each genotype.

For analysing feeding behavior in individual flies, animals were starved for 4 hours, before being placed in a Drosophila breeding chamber separated by a divider. Following a 5 min acclimation period, the divider was removed allowing the fly access to small drop of 5\% (wt/vol) glucose in in $\mathrm{ddH}_{2} \mathrm{O}$. The chambers were filmed in the ensuing 10 minute period, and the time spent in contact with food during this period was quantified post acquisition with $n=10-12$ for each genotype.

Nutrient rescue. An artificial diet chiefly consisting of a yeast-sugar medium was used for a nutritional rescue assay. The medium consisted of $80 \mathrm{~g} / \mathrm{l}$ yeast and $90 \mathrm{~g} / \mathrm{l}$ sucrose boiled with $1 \%$ agar, $4.8 \%$ propionic acid and 1.6\% methyl 4-hydroxybenzoate (all components from Sigma). Media with lower nutrient concentrations were made by keeping sucrose concentration constant but reducing yeast concentrations to $16 \mathrm{~g} / \mathrm{l}$ and $0 \mathrm{~g} / \mathrm{l}$, respectively. Up to 30 animals were transferred into vials containing the appropriate medium, and fly mortality was counted every 12 hours for the ensuing 10 days with a total of $n=251-323$ flies used per condition.

Nutrient-level assays. Four days after transfer to the restrictive temperature $\left(30^{\circ} \mathrm{C}\right)$, flies were collected and frozen at $-80{ }^{\circ} \mathrm{C}$ in groups of ten animals, and subsequently homogenized in $300 \mu \mathrm{PBS}+0.5 \%$ Tween-20 (PBST) using a TissueLyser LT (Qiagen). Next, $2 \mu 1$ was 
used to quantify protein content using the Bradford assay for subsequent normalization of nutrient levels. The samples were then heat-inactivated at $70^{\circ} \mathrm{C}$ for 5 minutes, centrifuged to pellet debris for 3 seconds at full speed, before the supernatant was transferred to fresh vials. For triacylglyceride measurements, $2 \mu$ of the supernatant was incubated with $4 \mu \mathrm{l}$ Triglyceride Reagent (Sigma, T2449) and $6 \mu \mathrm{PBST}$ at $37^{\circ} \mathrm{C}$ for 30 minutes; next, $20 \mu \mathrm{l}$ Free Glycerol Reagent (Sigma, F6428) was added, and the reaction was incubated at $37^{\circ} \mathrm{C}$ until color development occured (usually 30-60 min). Absorbance at $540 \mathrm{~nm}$ was then measured. For glycogen measurements, $1 \mu \mathrm{l}$ homogenate was mixed with $0.2 \mu \mathrm{l}(0.06 \mathrm{U})$ of low-glucose Amyloglucosidase (Sigma, A7420-5MG) in $9.8 \mu 1$ of PBST and incubated at $37^{\circ} \mathrm{C}$ for 30 minutes; Glucose Oxidase (GO) reagent ( $25 \mu \mathrm{l}$ ) from the Glucose (GO) Assay Kit (Sigma, GAGO20) was added to determine total glucose + glycogen. Another $2 \mu$ l homogenate was mixed with $25 \mu \mathrm{GO}$ reagent alone (without the Amyloglucosidase) to determine free glucose. To determine trehalose contents, $1 \mu 1$ supernatant was incubated with $0.5 \mu 1$ Trehalase from porcine kidney (Sigma, T8778) with $9.5 \mu \mathrm{PBST}$ and incubated at $37^{\circ} \mathrm{C}$ overnight. Glucose Oxidase (GO) reagent $(25 \mu \mathrm{l})$ was then added and incubated at $37^{\circ} \mathrm{C}$ to determine trehalose + free glucose. Free glucose readings were subtracted from measurements of glycogen + glucose and trehalose + glucose to obtain readings for glycogen and trehalose, respectively. All absorbances were measured using an EnSight multimode plate reader (PerkinElmer), and values were normalized to protein content.

To measure the lipid and glucose contents of excreta, 3 days after temperature shift, flies were incubated with $0.5 \%$ BPB-supplemented fly medium for an additional day for prefeeding. Then, groups of five flies were transferred to 2-ml tubes containing BPB food cast into the lid of the tube for an additional 24 hours at $30^{\circ} \mathrm{C}$. After carefully removing the animals and cutting of the lids, tubes were washed with $100 \mu \mathrm{l}$ of PBST, gently agitated with 
fresh lids and read at $540 \mathrm{~nm}$ absorbance for normalization. To measure the lipid and glucose contents of excreta, $10 \mu \mathrm{l}$ of extracts were used and quantified as described above.

Locomotor assays. To analyse long-term locomotor activity of individual flies, we used the Drosophila activity monitor system (TriKinetics). Individual 4-day old flies of the appropriate genotype were housed in 65-mm-long glass tubes containing food ( $5 \%$ sucrose and $2 \%$ agar in water) in one end and a cotton plug in the other, which were assembled into the monitoring system. The experiments were run in a behavioral incubator at $25{ }^{\circ} \mathrm{C}$ with 50 $60 \%$ humidity and a 12-hour:12-hour light-dark circadian rythm. The flies were allowed to acclimate for 14 hours prior to data acquisition. The activity of individual flies was then measured as the number of beam crossings per 10 minutes, which was calculated using pySolo software in combination with an in-house MATLAB script. The stereotypic early activity period (EAP) and late activity period (LAP) were used to assess significant changes in locomotor activity.

We also analyzed short-term parameters of locomotor activity using a custom-built activity monitor system. Flies $(\mathrm{n}=10-16)$ were isolated individually in Plexiglas chambers, and allowed to acclimate for 2 hours. Flies were then recorded at the same time for 5 minutes using a high-resolution digital camera. Following an experimental cycle, parameters such as activity traces, total distance traveled and the maximal response velocity for each individual fly was calculated in MATLAB.

Histochemistry. Adult brains, muscles, and fat bodies were dissected in PBS and fixed in 4\% PFA in PBS for 20 minutes. Next, tissues were washed twice in PBS, incubated with periodic acid solution (Merck \#395B-1KT) for 5 min, and washed twice in PBS. Samples were then stained with Schiff's reagent (Merck \#395B-1KT) for 15 minutes, washed twice in PBS, and 
mounted in 50\% glycerol in PBS. Images were acquired with an BX-51 microscope equipped with an digital camera (Olympus). Two independent experiments were conducted and gave the same results.

Starvation resistance. Flies were raised at the permissive temperature $\left(18^{\circ} \mathrm{C}\right)$ until 24 hours after eclosion, after which they were transferred to the restrictive temperature $\left(30^{\circ} \mathrm{C}\right)$ to disinhibit GAL4. Three days after transgene activation at $30{ }^{\circ} \mathrm{C}$, flies were transferred unto $1 \%$ non-nutritive agar and incubated at $30{ }^{\circ} \mathrm{C}$. Each vial was then observed every 8 hours until all flies $(n=213-288)$ were counted as dead.

Ramsay fluid secretion assay. Secretion assays were performed as described previously ${ }^{60}$. Intact Malpighian tubules from 7-day-old female flies were dissected in AHL and isolated into a $10 \mu 1$ drop of a 1:1 mixture of AHL and Drosophila saline. The tubules were left to secrete for approximately 30 minutes, with non-secreting tubules being replaced if necessary, to produce a set of 10-20 working tubules. A drop of secreted fluid was subsequently collected every 10 minutes, and the diameter was measured using an eyepiece graticule. The volume of each droplet was calculated as $4 \pi r^{3} / 3$, where $r$ is the radius of the droplet, and secretion rates were plotted against time. Secretion was measured under basal conditions in order to establish a steady rate of secretion, prior to stimulation with labeled or unlabeled Capa peptides, with an increase in fluid secretion rate being taken as an indication of a diuretic effect.

Statistics. Numerical data are presented as means \pm s.e.m. The normal (Gaussian) distribution of data were tested using D'Agostino-Pearsen omnibus normality test. A onetailed Student's $t$-test was used to evaluate the significance of the results between two 
samples. For multiple comparisons tests, a one-way ANOVA followed by Tukey's multiple comparisons of means was applied. Survival curves were assessed by the log-rank (MantelCox) test and conducted for each pairwise comparison. Significance levels of $\mathrm{P}<0.05(*)$, $\mathrm{P}<0.01(* *), \mathrm{P}<0.001(* * *)$ and $\mathrm{P}<0.0001(* * * *)$ were used in all tests. The statistical tests were performed using the data analysis program GraphPad Prism 8.0 (GraphPad Software Inc., CA, USA).

\section{AUTHOR CONTRIBUTIONS}

T.K., S.T. and K.A.H. designed and conceptualized the study. T.K., S.T., M.T.N, S.N. and K.A.H. performed the experiments and analyzed the data. K.A.H. wrote the manuscript with input from T.K .and S.T. All authors reviewed and edited the manuscript. K.A.H. produced the figures, directed the project and provided the funding.

\section{ACKNOWLEDGEMENTS}

We are grateful to Manfred Frasch, Achim Paululat, Diego Manuel and Dr. Jae Park, for the generous sharing of resources, as well as to Michael Texada for giving critical comments to the manuscript. Camilla Trang Vo and Christina Papamichail are thanked for helping with pupal weight and developmental timing quantifications. This work was supported by funding from the Villum Foundation (15365) and Danish Council for Independent research Natural Sciences (9064-00009B) to KAH. Additional funding was given by UKRI BBSRC (BB/P008097/1) to SD, JATD and ST as well as by Novo Nordisk Foundation (16OC0021270) to K.R. 


\section{REFERENCES}

1 Droujinine, I. A. \& Perrimon, N. Interorgan Communication Pathways in Physiology: Focus on Drosophila. Annual review of genetics 50, 539-570, doi:10.1146/annurev-genet-121415122024 (2016).

2 Castillo-Armengol, J., Fajas, L. \& Lopez-Mejia, I. C. Inter-organ communication: a gatekeeper for metabolic health. EMBO Rep 20, e47903, doi:10.15252/embr.201947903 (2019).

3 Bourque, C. W. Central mechanisms of osmosensation and systemic osmoregulation. Nat Rev Neurosci 9, 519-531, doi:10.1038/nrn2400 (2008).

4 Sternson, S. M. Hypothalamic survival circuits: blueprints for purposive behaviors. Neuron 77, 810-824, doi:10.1016/j.neuron.2013.02.018 (2013).

5 Koyama, T., Texada, M. J., Halberg, K. A. \& Rewitz, K. Metabolism and growth adaptation to environmental conditions in Drosophila. Cell Mol Life Sci, doi:10.1007/s00018-020-03547-2 (2020).

6 Dus, M. et al. Nutrient Sensor in the Brain Directs the Action of the Brain-Gut Axis in Drosophila. Neuron 87, 139-151, doi:10.1016/j.neuron.2015.05.032 (2015).

7 Jourjine, N., Mullaney, B. C., Mann, K. \& Scott, K. Coupled Sensing of Hunger and Thirst Signals Balances Sugar and Water Consumption. Cell 166, 855-866, doi:10.1016/j.cell.2016.06.046 (2016).

8 Oh, Y. et al. A glucose-sensing neuron pair regulates insulin and glucagon in Drosophila. Nature 574, 559-564, doi:10.1038/s41586-019-1675-4 (2019).

$9 \quad$ Predel, R. \& Wegener, C. Biology of the CAPA peptides in insects. Cell Mol Life Sci 63, 24772490, doi:10.1007/s00018-006-6187-3 (2006).

10 Kean, L. et al. Two nitridergic peptides are encoded by the gene capability in Drosophila melanogaster. American journal of physiology. Regulatory, integrative and comparative physiology 282, R1297-1307, doi:10.1152/ajpregu.00584.2001 (2002).

11 Wegener, C., Reinl, T., Jansch, L. \& Predel, R. Direct mass spectrometric peptide profiling and fragmentation of larval peptide hormone release sites in Drosophila melanogaster reveals tagma-specific peptide expression and differential processing. J Neurochem 96, 1362-1374 (2006).

12 Terhzaz, S. et al. Mechanism and function of Drosophila capa GPCR: a desiccation stressresponsive receptor with functional homology to human neuromedinU receptor. PLoS One 7, e29897 (2012).

13 Budhiraja, S. \& Chugh, A. Neuromedin U: physiology, pharmacology and therapeutic potential. Fundam Clin Pharmacol 23, 149-157, doi:10.1111/j.1472-8206.2009.00667.x (2009).

14 Duffy, J. B. GAL4 system in Drosophila: a fly geneticist's Swiss army knife. Genesis 34, 1-15 (2002).

15 McGuire, S. E., Mao, Z. \& Davis, R. L. Spatiotemporal gene expression targeting with the TARGET and gene-switch systems in Drosophila. SCi STKE 2004, pl6, doi:10.1126/stke.2202004pl6 (2004).

16 Halberg, K. A., Terhzaz, S., Cabrero, P., Davies, S. A. \& Dow, J. A. T. Tracing the evolutionary origins of insect renal function. Nat Commun 6 (2015).

17 Terhzaz, S. et al. Cell-specific inositol 1,4,5 trisphosphate 3-kinase mediates epithelial cell apoptosis in response to oxidative stress in Drosophila. Cell Signal 22, 737-748, doi:10.1016/j.cellsig.2009.12.009 (2010).

18 Zaffran, S., Astier, M., Gratecos, D. \& Sémériva, M. The held out wings (how) Drosophila gene encodes a putative RNA-binding protein involved in the control of muscular and cardiac activity. Development (Cambridge, England) 124, 2087-2098 (1997). 
19 Dutta, D. et al. Regional Cell-Specific Transcriptome Mapping Reveals Regulatory Complexity in the Adult Drosophila Midgut. Cell reports 12, 346-358, doi:10.1016/j.celrep.2015.06.009 (2015).

20 Prendergast, C. E., Morton, M. F., Figueroa, K. W., Wu, X. \& Shankley, N. P. Speciesdependent smooth muscle contraction to Neuromedin $U$ and determination of the receptor subtypes mediating contraction using NMU1 receptor knockout mice. British journal of pharmacology 147, 886-896, doi:10.1038/sj.bjp.0706677 (2006).

21 Wayland, M. T. et al. Spotting the differences: probing host/microbiota interactions with a dedicated software tool for the analysis of faecal outputs in Drosophila. Journal of insect physiology 69, 126-135, doi:10.1016/j.jinsphys.2014.05.023 (2014).

22 Cognigni, P., Bailey, A. P. \& Miguel-Aliaga, I. Enteric neurons and systemic signals couple nutritional and reproductive status with intestinal homeostasis. Cell Metab 13, 92-104, doi:10.1016/j.cmet.2010.12.010 (2011).

23 King, A. N. et al. A Peptidergic Circuit Links the Circadian Clock to Locomotor Activity. Current biology : CB 27, 1915-1927.e1915, doi:10.1016/j.cub.2017.05.089 (2017).

24 O'Donnell, M. J. \& Maddrell, S. H. Fluid reabsorption and ion transport by the lower Malpighian tubules of adult female Drosophila. J Exp Biol 198, 1647-1653 (1995).

25 Bielefeldt, K., Tuteja, A. \& Nusrat, S. Disorders of gastrointestinal hypomotility. F1000Res 5, F1000 Faculty Rev-1897, doi:10.12688/f1000research.8658.1 (2016).

26 Dubreuil, R. R. Copper cells and stomach acid secretion in the Drosophila midgut. Int J Biochem Cell Biol 36, 745-752 (2004).

27 Hanada, T. et al. Central actions of neuromedin U via corticotropin-releasing hormone. Biochem Biophys Res Commun 311, 954-958, doi:10.1016/j.bbrc.2003.10.098 (2003).

28 Ivanov, T. R., Lawrence, C. B., Stanley, P. J. \& Luckman, S. M. Evaluation of neuromedin U actions in energy homeostasis and pituitary function. Endocrinology 143, 3813-3821, doi:10.1210/en.2002-220121 (2002).

29 Dietrich, M. O., Zimmer, M. R., Bober, J. \& Horvath, T. L. Hypothalamic Agrp neurons drive stereotypic behaviors beyond feeding. Cell 160, 1222-1232, doi:10.1016/j.cell.2015.02.024 (2015).

30 Lee, G. \& Park, J. H. Hemolymph sugar homeostasis and starvation-induced hyperactivity affected by genetic manipulations of the adipokinetic hormone-encoding gene in Drosophila melanogaster. Genetics 167, 311-323, doi:10.1534/genetics.167.1.311 (2004).

31 Zandawala, M. et al. Modulation of Drosophila post-feeding physiology and behavior by the neuropeptide leucokinin. PLoS Genet 14, e1007767, doi:10.1371/journal.pgen.1007767 (2018).

32 Wegener, C. \& Nassel, D. R. Peptide-induced $\mathrm{Ca}(2+)$ movements in a tonic insect muscle: effects of proctolin and periviscerokinin-2. J Neurophysiol 84, 3056-3066, doi:10.1152/jn.2000.84.6.3056 (2000).

33 Eckert, M., Predel, R. \& Gundel, M. Periviscerokinin-like immunoreactivity in the nervous system of the American cockroach. Cell and Tissue Research 295, 159-170, doi:10.1007/s004410051222 (1999).

34 Huesmann, G. R. et al. Amino acid sequence of CAP2b, an insect cardioacceleratory peptide from the tobacco hawkmoth Manduca sexta. FEBS Lett 371, 311-314, doi:10.1016/00145793(95)00929-4 (1995).

35 Yamada, T., Habara, O., Kubo, H. \& Nishimura, T. Fat body glycogen serves as a metabolic safeguard for the maintenance of sugar levels in Drosophila. Development 145, doi:10.1242/dev.158865 (2018).

36 Maack, C. \& O'Rourke, B. Excitation-contraction coupling and mitochondrial energetics. Basic Res Cardiol 102, 369-392, doi:10.1007/s00395-007-0666-z (2007). 
37 Bai, J. et al. RNA interference screening in Drosophila primary cells for genes involved in muscle assembly and maintenance. Development (Cambridge, England) 135, 1439-1449, doi:10.1242/dev.012849 (2008).

38 Vallejo-Illarramendi, A., Toral-Ojeda, I., Aldanondo, G. \& Lopez de Munain, A. Dysregulation of calcium homeostasis in muscular dystrophies. Expert Rev Mol Med 16, e16, doi:10.1017/erm.2014.17 (2014).

39 Terhzaz, S. et al. Insect capa neuropeptides impact desiccation and cold tolerance. Proceedings of the National Academy of Sciences 112, 2882-2887, doi:10.1073/pnas.1501518112 (2015).

40 Masuyama, K., Zhang, Y., Rao, Y. \& Wang, J. W. Mapping neural circuits with activitydependent nuclear import of a transcription factor. Journal of neurogenetics 26, 89-102, doi:10.3109/01677063.2011.642910 (2012).

41 Mattila, J. \& Hietakangas, V. Regulation of Carbohydrate Energy Metabolism in Drosophila melanogaster. Genetics 207, 1231-1253, doi:10.1534/genetics.117.199885 (2017).

42 Texada, M. J. et al. Autophagy-Mediated Cholesterol Trafficking Controls Steroid Production. Dev Cell 48, 659-671.e654, doi:10.1016/j.devcel.2019.01.007 (2019).

43 Olds, W. H. \& Xu, T. Regulation of food intake by mechanosensory ion channels in enteric neurons. Elife 3, doi:10.7554/eLife.04402 (2014).

44 Al-Anzi, B. et al. The leucokinin pathway and its neurons regulate meal size in Drosophila. Curr Biol 20, 969-978, doi:10.1016/j.cub.2010.04.039 (2010).

45 Terhzaz, S. et al. Insect capa neuropeptides impact desiccation and cold tolerance. Proc Natl Acad Sci U S A 112, 2882-2887, doi:10.1073/pnas.1501518112 (2015).

46 Halberg, K. A., Terhzaz, S., Cabrero, P., Davies, S. A. \& Dow, J. A. Tracing the evolutionary origins of insect renal function. Nat Commun 6, 6800, doi:10.1038/ncomms7800 (2015).

47 Benguettat, O. et al. The DH31/CGRP enteroendocrine peptide triggers intestinal contractions favoring the elimination of opportunistic bacteria. PLoS Pathog 14, e1007279, doi:10.1371/journal.ppat.1007279 (2018).

48 Miguel-Aliaga, I., Jasper, H. \& Lemaitre, B. Anatomy and Physiology of the Digestive Tract of Drosophila melanogaster. Genetics 210, 357-396, doi:10.1534/genetics.118.300224 (2018).

49 Lemaitre, B. \& Miguel-Aliaga, I. The digestive tract of Drosophila melanogaster. Annual review of genetics 47, 377-404, doi:10.1146/annurev-genet-111212-133343 (2013).

50 LaJeunesse, D. R., Johnson, B., Presnell, J. S., Catignas, K. K. \& Zapotoczny, G. Peristalsis in the junction region of the Drosophila larval midgut is modulated by DH31 expressing enteroendocrine cells. BMC Physiol 10, 14, doi:10.1186/1472-6793-10-14 (2010).

51 Bolukbasi, E. et al. Intestinal Fork Head Regulates Nutrient Absorption and Promotes Longevity. Cell Rep 21, 641-653, doi:10.1016/j.celrep.2017.09.042 (2017).

52 Woudstra, T. \& Thomson, A. B. Nutrient absorption and intestinal adaptation with ageing. Best practice \& research. Clinical gastroenterology 16, 1-15, doi:10.1053/bega.2001.0262 (2002).

53 Riemensperger, T. et al. Behavioral consequences of dopamine deficiency in the Drosophila central nervous system. Proc Natl Acad Sci U S A 108, 834-839, doi:10.1073/pnas.1010930108 (2011).

54 Cannell, E. et al. The corticotropin-releasing factor-like diuretic hormone 44 (DH44) and kinin neuropeptides modulate desiccation and starvation tolerance in Drosophila melanogaster. Peptides 80, 96-107, doi:10.1016/j.peptides.2016.02.004 (2016).

55 Zhang, W. et al. Neuromedin U suppresses glucose-stimulated insulin secretion in pancreatic beta cells. Biochem Biophys Res Commun 493, 677-683, doi:10.1016/j.bbrc.2017.08.132 (2017).

56 Liu, L. et al. Drosophila hygrosensation requires the TRP channels water witch and nanchung. Nature 450, 294-298, doi:10.1038/nature06223 (2007). 
57 Gáliková, M., Dircksen, H. \& Nässel, D. R. The thirsty fly: Ion transport peptide (ITP) is a novel endocrine regulator of water homeostasis in Drosophila. PLoS genetics 14, e1007618e1007618, doi:10.1371/journal.pgen.1007618 (2018).

$58 \quad$ Lin, S. et al. Neural correlates of water reward in thirsty Drosophila. Nat Neurosci 17, 15361542, doi:10.1038/nn.3827 (2014).

59 Halberg, K. A. \& Mobjerg, N. First evidence of epithelial transport in tardigrades: a comparative investigation of organic anion transport. Journal of Experimental Biology 215, 497-507, doi:10.1242/jeb.065987 (2012).

60 Halberg, K. A. et al. The cell adhesion molecule Fasciclin2 regulates brush border length and organization in Drosophila renal tubules. Nat Commun 7, doi:10.1038/ncomms11266 (2016).

61 Rosay, P. et al. Cell-type specific calcium signalling in a Drosophila epithelium. Journal of Cell Science 110 ( Pt 15), 1683-1692 (1997).

62 Dutta, D. et al. Regional Cell-Specific Transcriptome Mapping Reveals Regulatory Complexity in the Adult Drosophila Midgut. Cell reports 12, 346-358, doi:10.1016/j.celrep.2015.06.009 (2015). 
Figure legends
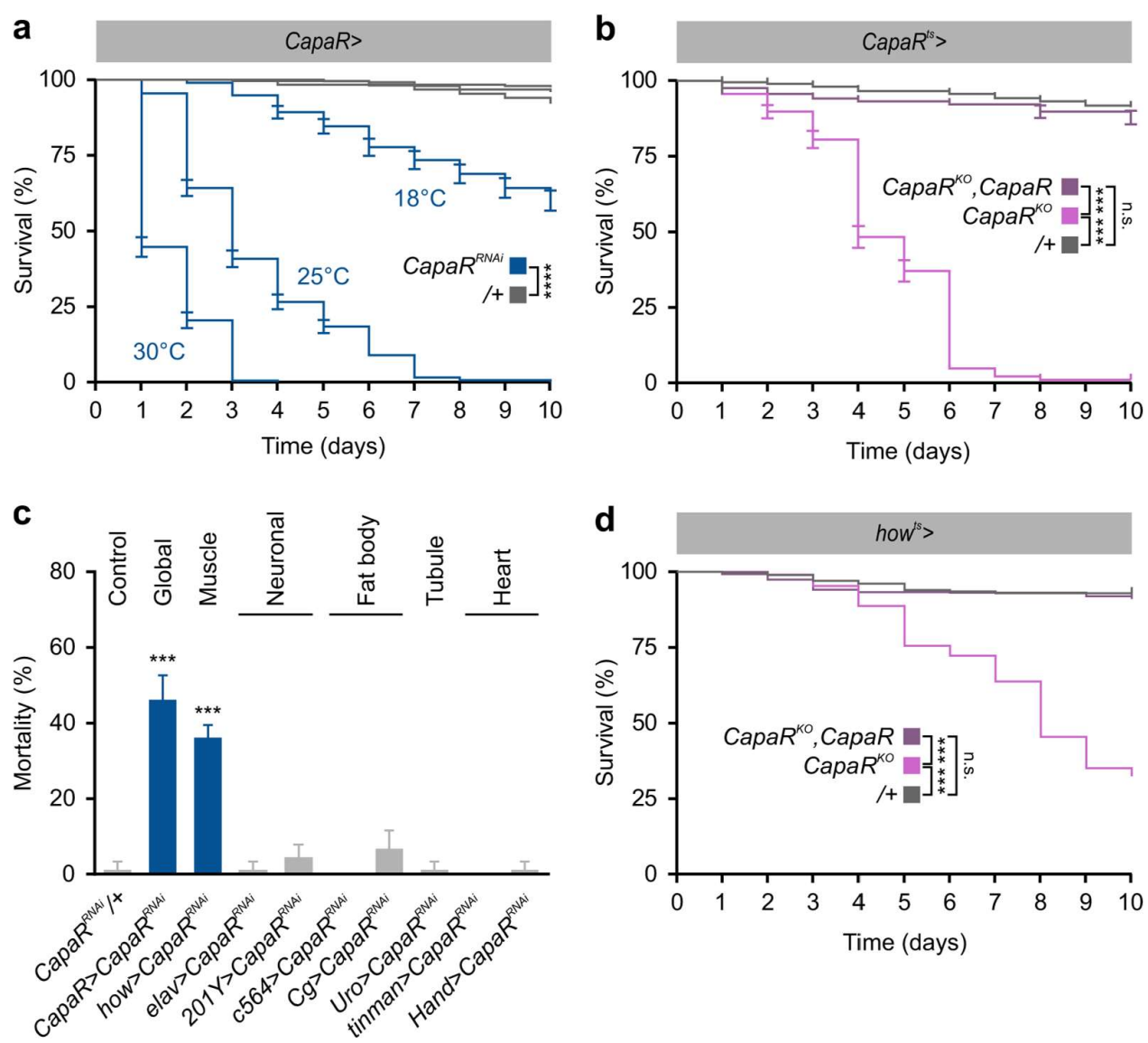

Figure 1

Figure 1. Loss of CapaR decreases adult survival. a. Global knockdown of CapaR $\left(\right.$ CapaR $>2 x$ CapaR $\left.^{R N A i}\right)$ results in strong mortality shortly after adult emergence. Lethality correlates with increased temperature due to the thermal sensitivity of the GAL4/UASsystem. Median survival for male flies was 10,3 , and 1 days at $18^{\circ} \mathrm{C}(\mathrm{n}=213), 25^{\circ} \mathrm{C}(\mathrm{n}=293)$ and $30^{\circ} \mathrm{C}(\mathrm{n}=232)$, respectively (log-rank test). b. Adult-specific CapaR knockout $\left(\right.$ CapaR $^{t s}>$ CapaR $\left.^{K O}\right)$ induces significant mortality of male flies $(\mathrm{n}=498)$ compared to controls (log-rank test; $\mathrm{n}=436$;). This lethality was fully rescued in flies additionally carrying a $U A S$ CapaR transgene $\left(\right.$ CapaR $^{t s}>$ CapaR $^{K O}$, CapaR; $\left.\mathrm{n}=451\right)$. c. Knockdown of CapaR in whole animals $($ CapaR $>$ ) or exclusively in muscles (how $>$ ), but not in neurons (elav $>$ or 201Y), fat 
body $(C g>)$, Malpighian tubules $($ Uro $>$ ) or heart (tinman $>$ or hand $>$ ), cause significant male fly mortality (one-way-ANOVA; n=60). d. Muscle-specific CapaR knockout $\left(\right.$ how $^{t s}>$ CapaR $\left.^{K O}\right)$ recapitulates the lethality phenotype with knockout flies $(\mathrm{n}=793)$ showing significantly higher mortality compared to control (log-rank test; $n=723)$, and survival is completely rescued in flies co-expressing a receptor transgene $\left(h_{o w}^{t s}>\operatorname{CapaR}^{K O}\right.$, CapaR; $\mathrm{n}=600$ ). 

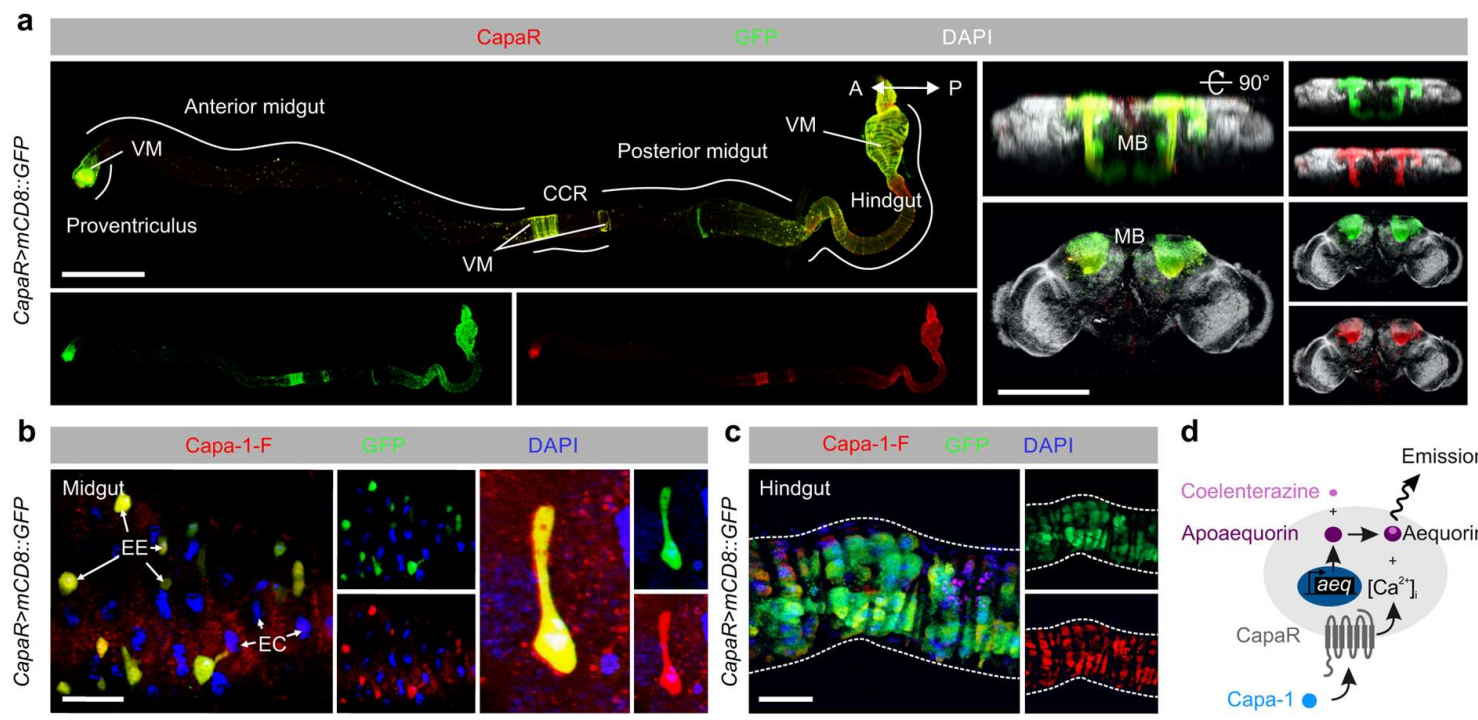

d
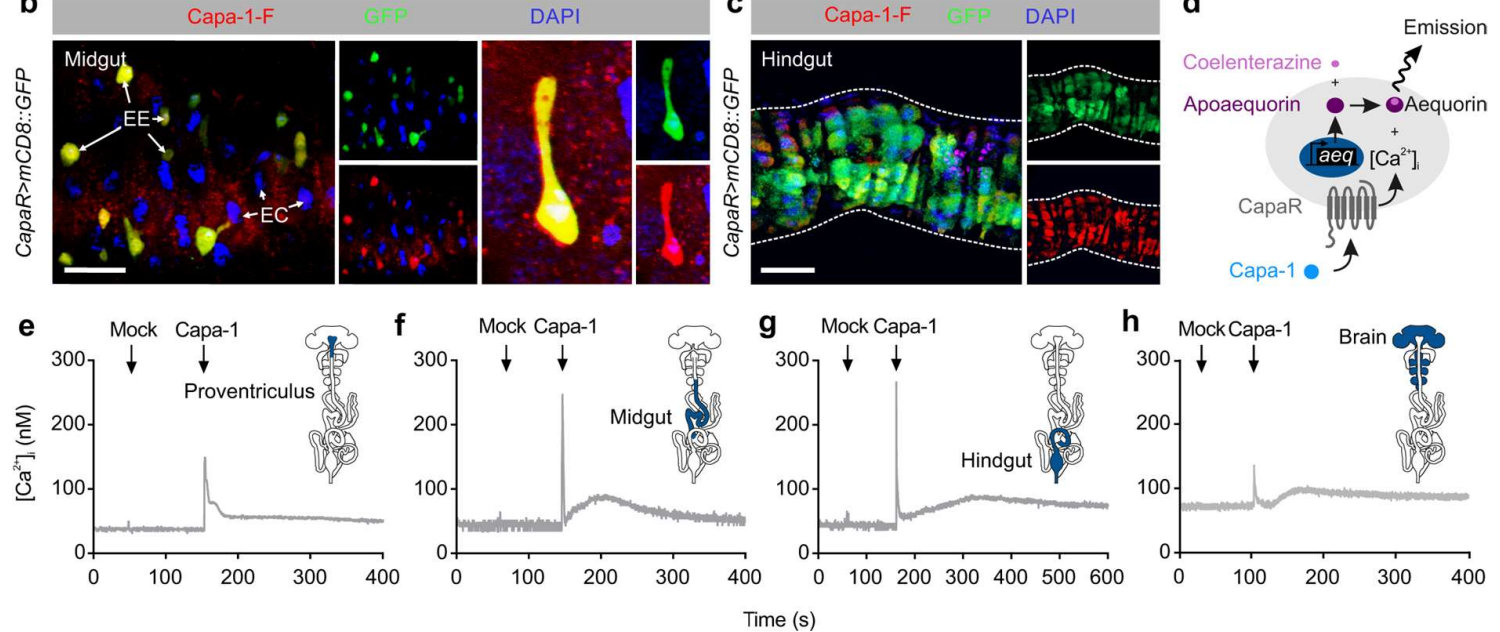

Figure 2

Figure 2. Global CapaR receptor mapping in adult Drosophila. a. Co-immunolabeling of the CNS and the intestine from $C a p a R>G F P$ flies with a custom generated CapaR antibody. Consistently, we observed an overlap between GFP (green) and CapaR (red) immunoreactivity in the mushroom body and neuronal somata of the CNS, as well as in the visceral muscles and enteroendocrine cells restricted to distinct portions of the gut. b-c. Application of fluorophore-labelled Capa-1 (Capa-1-F, red) on the intestine shows specific and displaceable binding to $C a p a R>G F P$ (green) positive enteroendocrine cells and visceral muscles. d. Principle of in vivo aequorin luminescence-based functional calcium assay in Drosophila tissues. e-h. Real-time changes in intracellular $\mathrm{Ca}^{2+}$ concentration of tissues natively dissected from flies expressing a UAS-apoaequorin transgene under the control of CapaR $>$. Cytosolic $\left[\mathrm{Ca}^{2+}\right]^{\mathrm{i}}$ levels $(\mathrm{nM})$ in e. proventriculus, f. midgut, g. hindgut and h. brain upon Capa-1 stimulation $\left(10^{-7} \mathrm{M}\right) . \mathrm{MB}$, mushroom body; EE, enteroendocrine cells; EC, enterocytes; CCR, copper-cell region. 


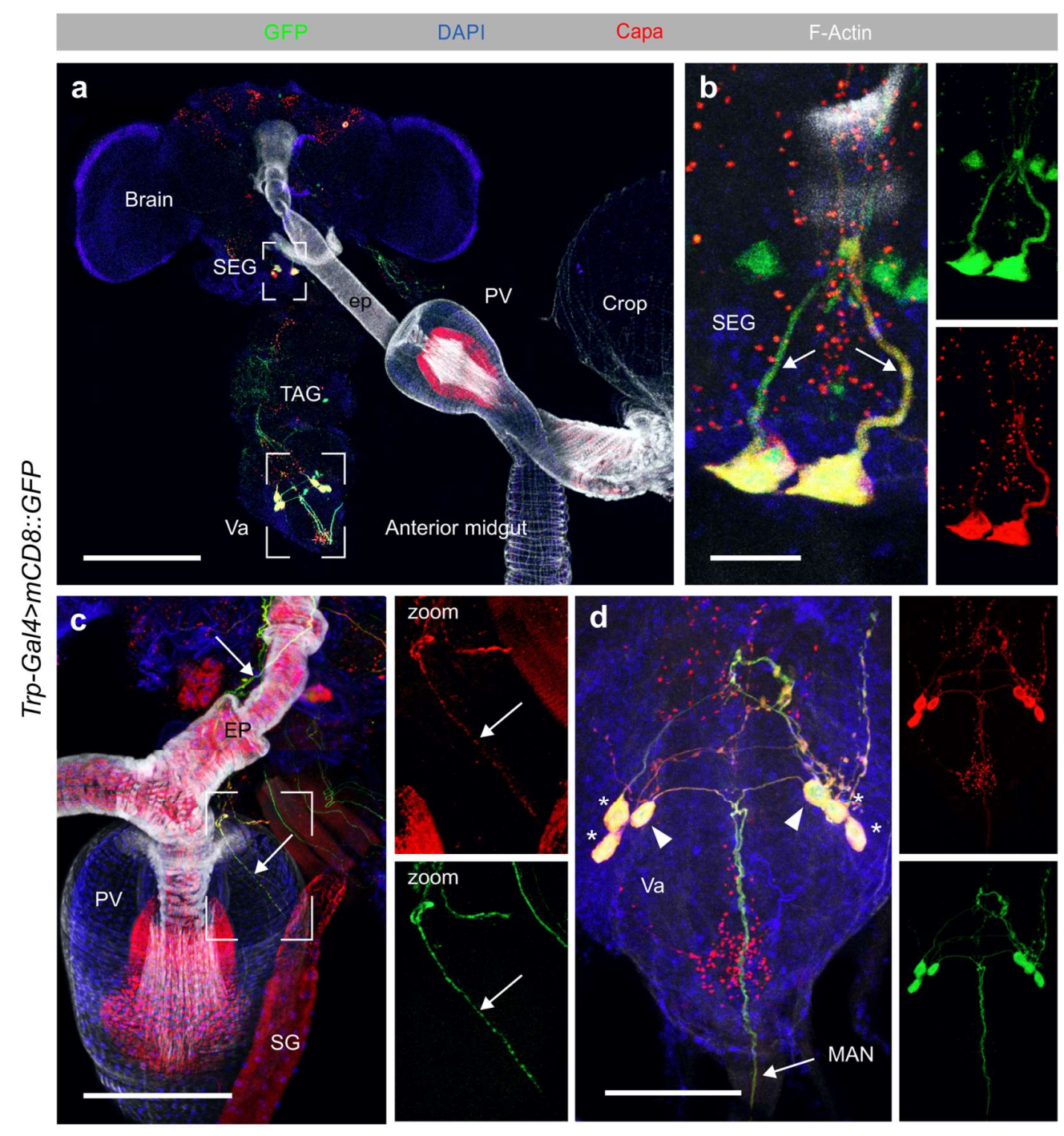

Figure 3

Figure 3. Anatomy of Capa-producing neurons in adult Drosophila. a-d. The CNS and associated tissues showing Trp $>U A S-m C D 8:: G F P$ (green) counterstained with an anti-Capa antibody (red), F-Actin (white) and DAPI (blue) in maximum projected z-stack confocal images a. GFP expression and anti-Capa immunoreactivity co-localize in four pairs of $\mathrm{Capa}^{+}$ neurons; one pair in the SEG and three pairs in the TAG. Scale bar, $200 \mu \mathrm{m}$. b. The SEG neurons extend dorsally. Scale bar, $20 \mu \mathrm{m}$. c. The SEG neurons also extend along the esophagus to innervate the proventriculus. Scale bar, $50 \mu \mathrm{m}$. d. The two anterior pairs $\left(^{*}\right)$ of Va neurons send axons to the dorsal neural sheath, while the posterior pair (triangles) projects 
bioRxiv preprint doi: https://doi.org/10.1101/2020.07.24.219592; this version posted July 25, 2020. The copyright holder for this preprint (which

was not certified by peer review) is the author/funder, who has granted bioRxiv a license to display the preprint in perpetuity. It is made available under aCC-BY-NC-ND 4.0 International license.

into the median abdominal nerve and form neurohemal release sites. Scale bar, $50 \mu \mathrm{m}$. EP, esophagus; MAN, median abdominal nerve; PV, proventriculus; SEG, subesophageal ganglion; SG, salivary gland; TAG, thoracicoabdominal ganglion; Va, ventroabdominal. 

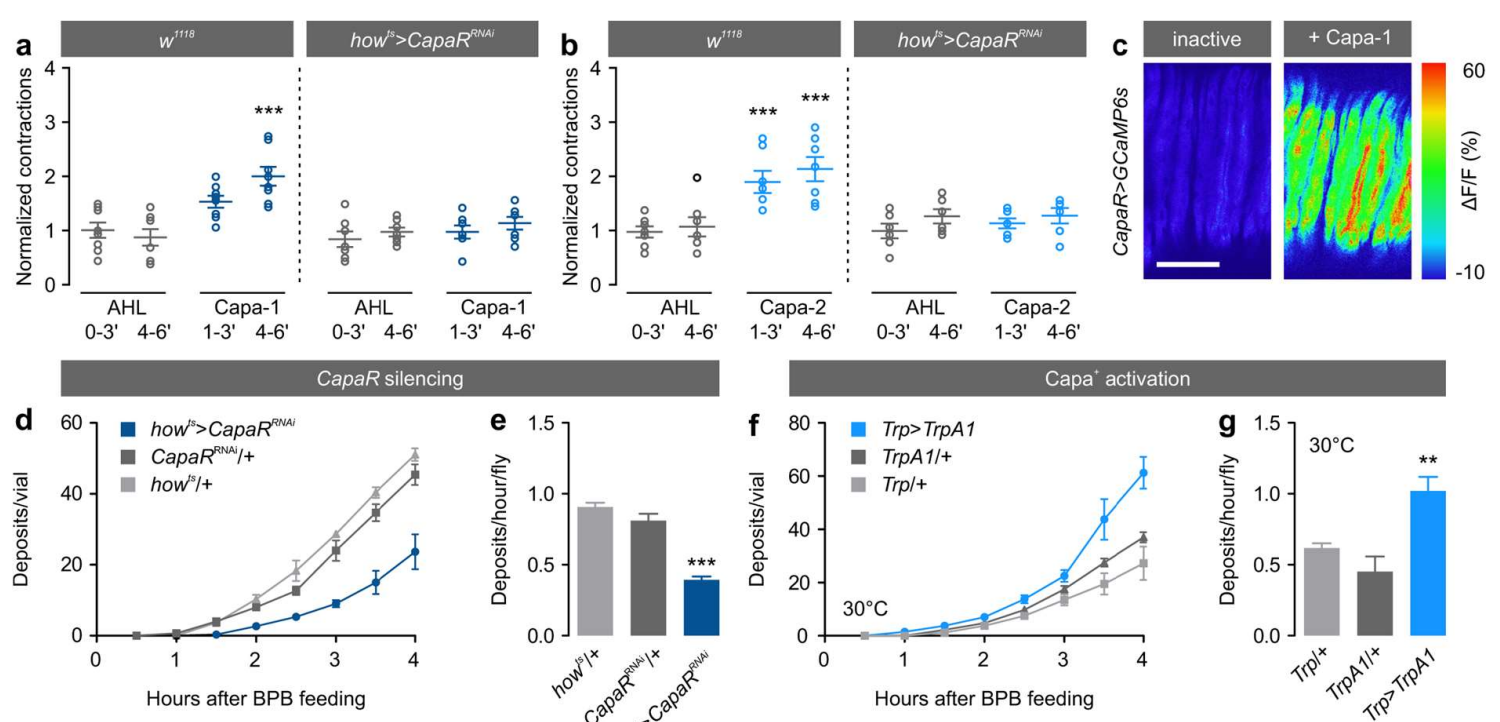

h
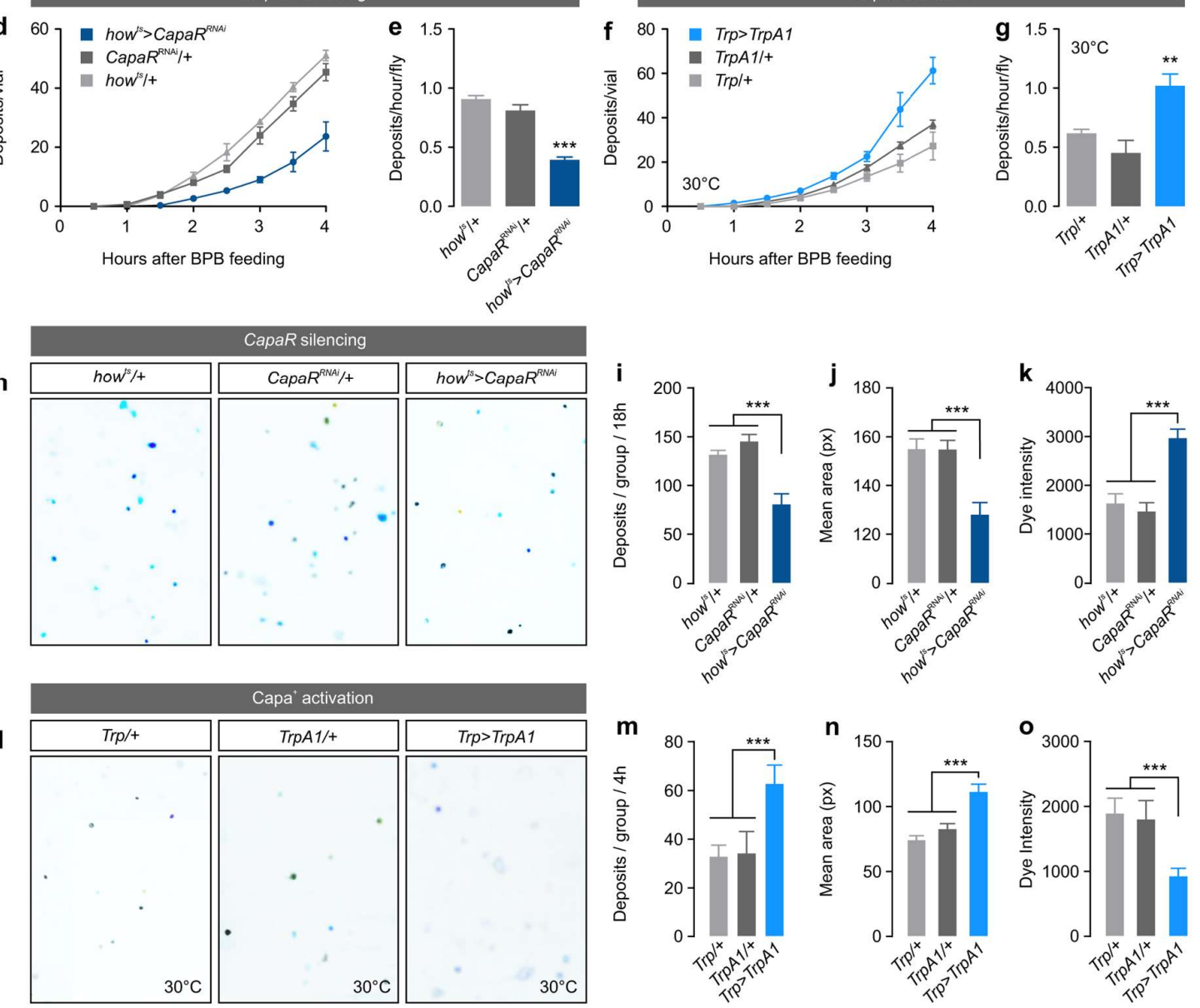

Figure 4

Figure 4. Capa modulates intestinal contractions and excretory physiology. a-b.

Spontaneous gut contractions from control and muscle-specific CapaR knockdown flies

$\left(h o w^{t s}>C_{a p a R}^{R N A i}\right)$ in response to AHL and AHL containing a. Capa-1 or b. Capa-2 at a concentration of $10^{-7} \mathrm{M}$. Grey values indicate exposures to AHL alone, whereas colored values refer to exposures to $\mathrm{AHL}+\mathrm{Capa}-1$ or Capa-2, respectively. $\Delta$ Contractions were calculated by normalizing the number of contractions during Capa-1/-2 application over those in the control (one-way-ANOVA; $\mathrm{n}=6-8$ ). $\mathbf{c}$. Inactive and activated circular visceral muscles 
in guts from flies carrying $U A S$-GCaMP6s driven by CapaR> following Capa-1 stimulation.

d. Accumulated number of deposits, and e. the number of deposits/hour/fly in musclespecific CapaR silenced flies $\left(h_{o w} w^{t s}>C_{a p a R^{R N A i}}\right)$ compared to parental controls (one-wayANOVA; $n=3$ vials with 20 flies per vial). f. Accumulated number of deposits, g. and the number of deposits/hour/fly in flies with artificial activation of the $\mathrm{Capa}^{+}$neurons by ectopic expression of the heat-sensitive $\operatorname{TrpA1}$ channel (Trp>TrpA1) compared to control flies at $30^{\circ} \mathrm{C}$ (one-way-ANOVA; $\mathrm{n}=3$ vials with 20 flies per vial). $\mathbf{h}, \mathbf{l}$. Representative fecal output profiles from the different genetic backgrounds fed on BPB-labelled food. Muscle-specific silencing of Capa signaling $\left(\right.$ how $^{t s}>\operatorname{CapaR}^{R N A i}$ ) results in i. fewer, j. smaller, and k. more concentrated BPB-labelled deposits compared to the parental controls (one-way-ANOVA; n=17-19 Petri dishes with 10 flies per dish). m. Conversely, artificial overactivation of Capaproducing neurons using a $U A S$-TrpA1 transgene induced excretion of more, n. larger, and $\mathbf{o .}$ lighter deposits compared to flies carrying each transgene alone (one-way-ANOVA; $n=5-7$ Petri dishes with 10 flies per dish). 
a

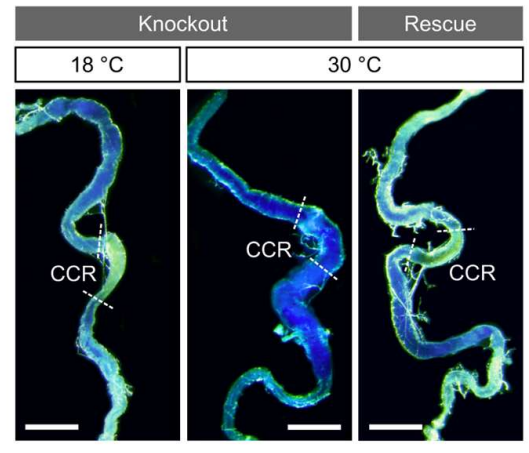

b $\quad$ CapaR ${ }^{\mathrm{RNAi}} /+$

now $>$ CapaR ${ }^{\text {enat }}$

25 -

$20-$

$\frac{\vec{d}}{\omega} 15-$

응 $10-$

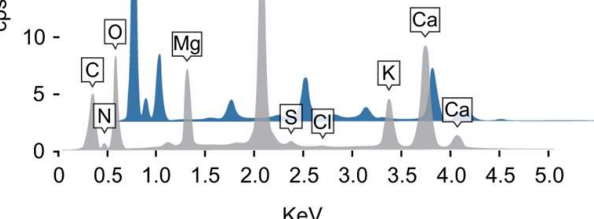

C

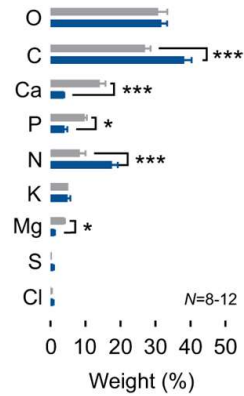

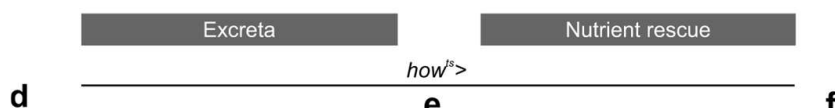
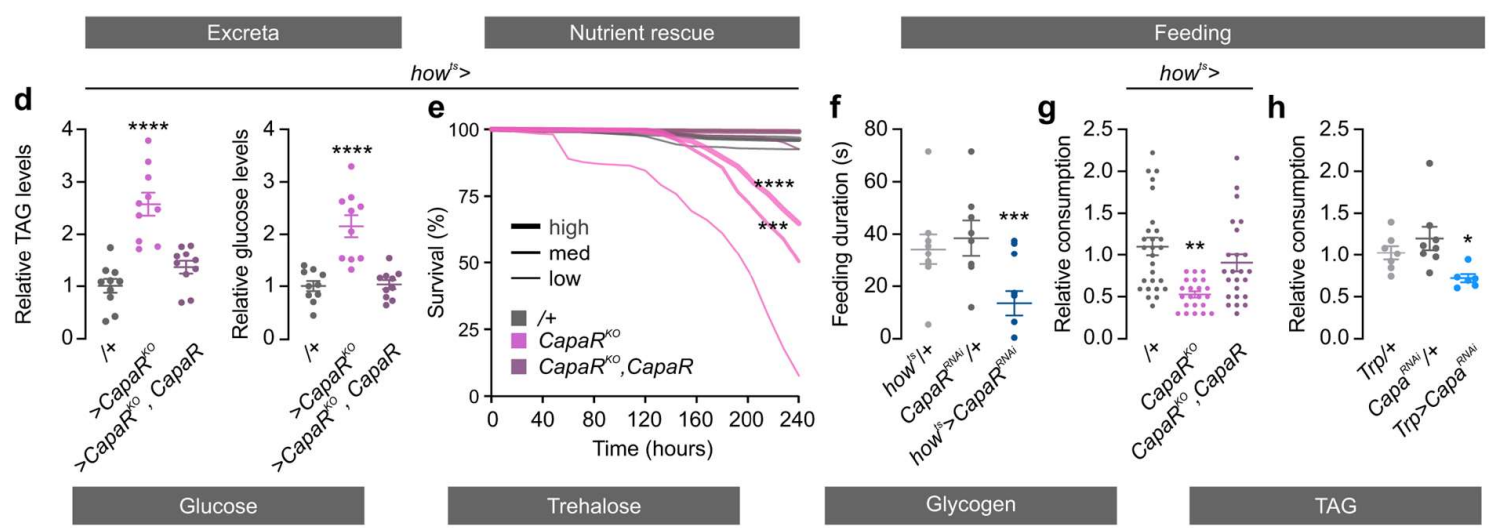

h
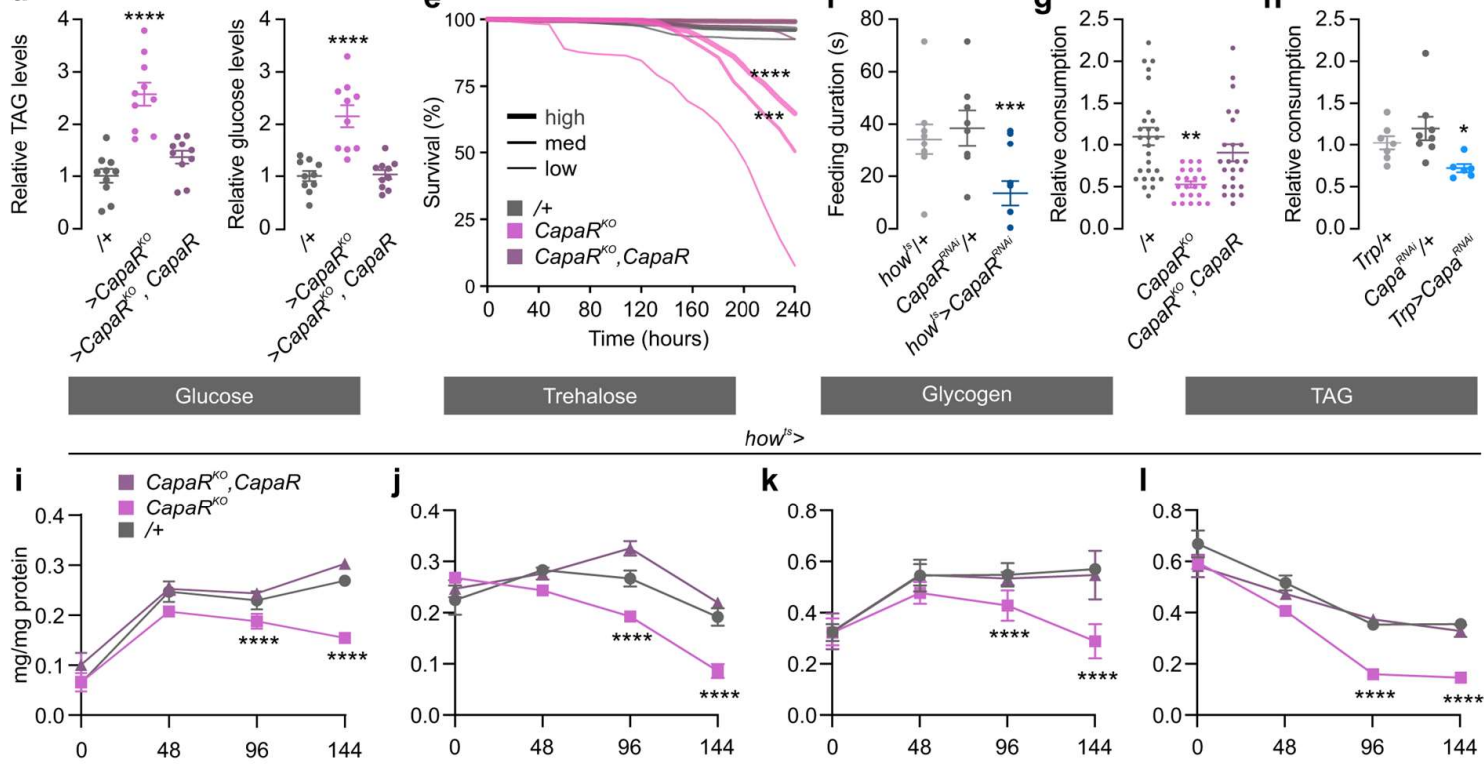

how ${ }^{15}>$
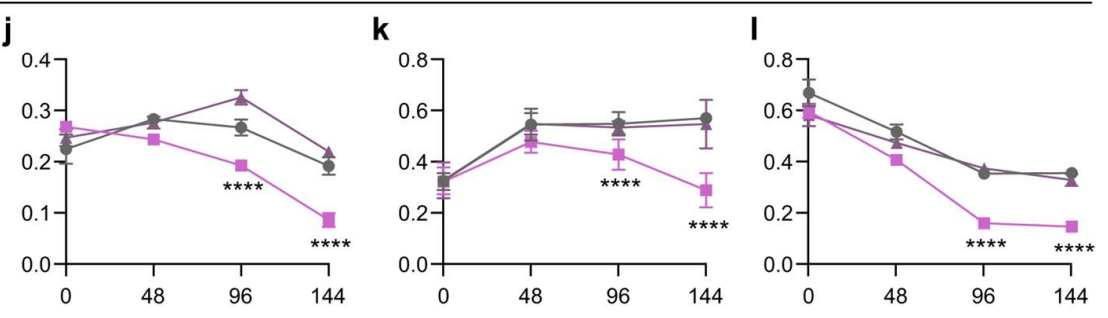

Figure 5

Figure 5. CapaR deletion in visceral muscles causes systemic metabolic imbalance. a.

Dissected intestinal tracts from flies fed with food supplemented with the $\mathrm{pH}$ indicator bromophenol blue. A complete loss of acidity in the copper-cell region (CCR) of the midgut was observed, as evident from the absence of yellowish color, exclusively in $h o w^{t s}>\operatorname{CapaR}^{K O}$ flies kept at the restrictive temperature $\left(30^{\circ} \mathrm{C}\right)$. This was not detected in animals kept at the permissive temperature $\left(18^{\circ} \mathrm{C}\right)$ or in rescue flies kept at $30^{\circ} \mathrm{C}$ additionally carrying a $U A S$ CapaR transgene $\left(\right.$ how $^{t s}>$ CapaR $^{K O}$, CapaR $)$. b. Elemental composition and c. percentage of weight element in the excreta from visceral muscle-specific CapaR-silenced flies (how $>$ CapaR ${ }^{R N A i}$ ) using scanning electron microscopy coupled with wave-dispersive X-ray 
analyses (unpaired $t$-test; $\mathrm{n}=8-12$ ). d. Relative amounts of undigested triacylglyceride (TAG) and glucose levels in excreta from control (/+), knockout $\left(\right.$ how $\left.^{t s}>\operatorname{CapaR}^{K O}\right)$ and rescue $\left(\right.$ how $^{\text {ts }}>$ CapaR $^{\text {KO, }}$ CapaR) flies raised on standard medium (one-way-ANOVA; $\mathrm{n}=10$ ). e. Flies kept on media with low, medium and high nutrient concentrations show that higher nutrient concentrations reduce mortality in $\operatorname{how}^{t s}>\operatorname{CapaR}^{K O}$ (low, n=325; med, n=323; high, $\mathrm{n}=325$ ) flies, with both control (low, $n=272$; med, $n=271$; high, $n=273$ ) and rescue (low, $n=251$; med, $\mathrm{n}=250$; high, $\mathrm{n}=253$ ) flies unaffected by the different nutrient dense diets (log-rank test). $\mathbf{f}$. Feeding duration was significantly decreased in CapaR knockdown animal $\left(\right.$ how ${ }^{t s}>$ CapaR $^{R N A i}$ ) compared to parental controls. g. Relative food intake in CapaR deficient $\left(\right.$ how $\left.^{t s}>\operatorname{CapaR}^{K O}\right)$ flies $(\mathrm{n}=8)$ and h. Capa silenced $\left(\operatorname{Trp}>\operatorname{Capa}^{R N A i}\right)$ flies (one-way ANOVA; n=6-8). i-l. Quantification of i. glucose $\mathbf{j}$. trehalose, k. glycogen and l. TAG levels following 48, 96 and 144 hours of adult-specific transgene activation (one-way ANOVA; $\mathrm{n}=10$ ). 

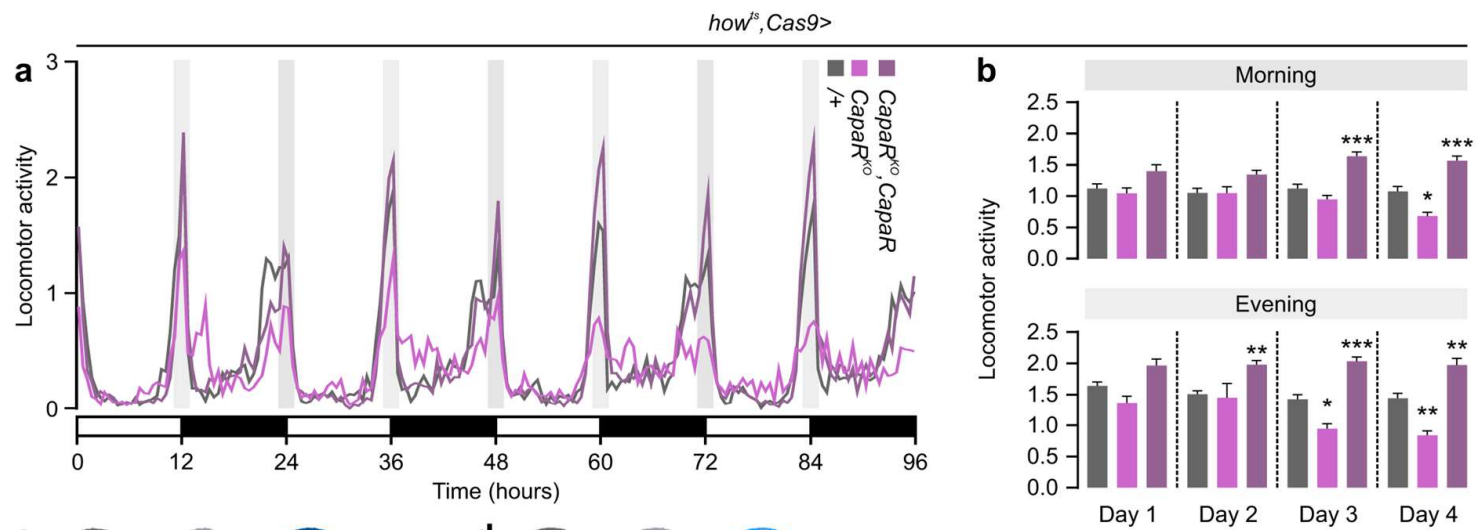

C
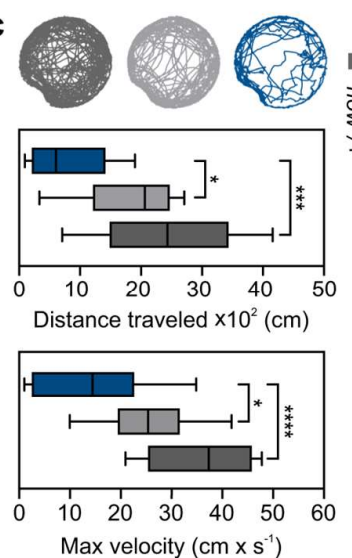

d
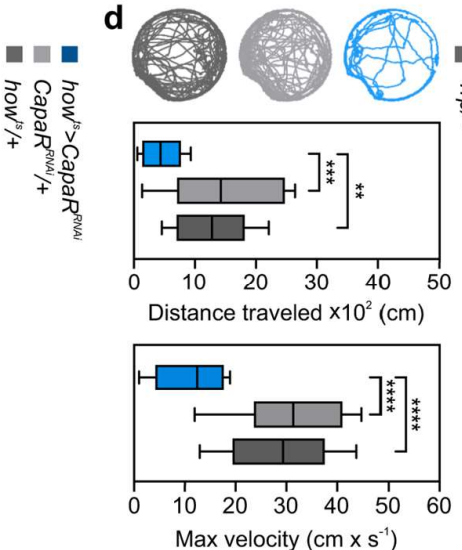

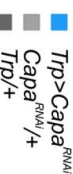

Skeletal muscle
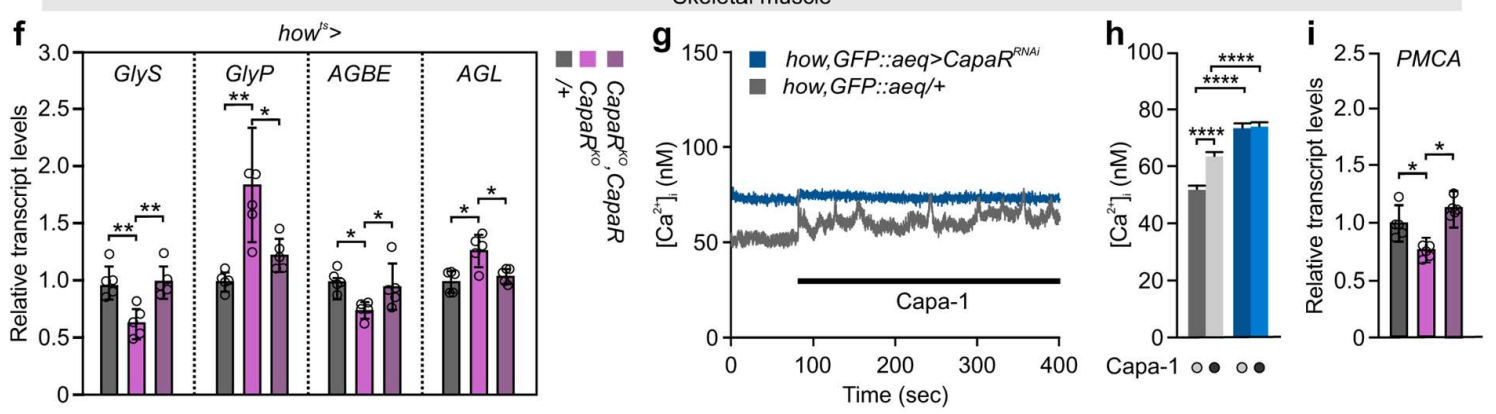

Figure 6

Figure 6. Muscle-specific CapaR elimination induces locomotor defects. a. Locomotor activity of individual control (/+; n=32), muscle-specific CapaR knockout $\left(\right.$ how $^{\text {ts }}>$ CapaR $^{\text {KO }}$; $\mathrm{n}=20)$ and rescue $\left(\right.$ how $^{t s}>\operatorname{CapaR}^{K O}$, CapaR; $\left.\mathrm{n}=32\right)$ flies exposed to 12-hour:12-hour, lightdark (LD) cycles for $96 \mathrm{~h}$. The experiment was repeated twice with the same results. $\mathbf{b}$.

Stereotypic morning and evening activity peaks measured over the experimental period (oneway ANOVA). c.-d. Representative activity traces of video-tracked individual flies with targeted CapaR silencing in muscles $\left(\mathrm{n}=10-16\right.$; how $^{t s}>$ CapaR $\left.^{R N A i}\right)$ or Capa knockdown in 
$\mathrm{Capa}^{+}$neurons (n=10-16; $\operatorname{Tr} p>\mathrm{Capa}^{R N A i}$ ) relative to control flies, including quantifications of distance traveled and max velocity (one-way ANOVA). e. Periodic Acid-Schiff (PAS) staining in the brain, muscle, and fat body showed depleted carbohydrate stores in these tissues of $h o w^{t s}>\operatorname{CapaR}^{K O}$ flies relative to control, with partial rescue in flies additionally carrying a CapaR transgene (how ${ }^{t s}>$ CapaR $^{K O}$,CapaR). f. Transcript levels of GlyS, GlyP, $A G B E$ and $A G L$ relative to $R p L 32$ in skeletal muscles (thorax samples) from muscle-specific CapaR knockout $\left(\right.$ how $\left.^{t s}>\operatorname{CapaR}^{K O}\right)$ and rescue $\left(h o w^{t s}>\operatorname{CapaR}^{K O}\right.$, CapaR $)$ flies. Data are expressed as mean fold change compared to control \pm s.e.m. (one-way ANOVA; $n=5$ ). g. Stereotypic cytosolic calcium traces in muscles expressing the aequorin-based bioluminescent $\mathrm{Ca}^{2+}$-indicator (how, GFP::aeq, grey line), and the effect of attenuating CapaR expression (how, GFP::aeq $>$ CapaR $^{R N A i}$, blue line) in muscles stimulated with $10^{-7} \mathrm{M}$ of Capa-1 peptide. h. Bar graphs of basal and stimulated cytosolic calcium levels in how;GFP::aequorin $>C_{a p a R^{R N A i}}$ and how;GFP::aequorin/+ control samples Data are expressed as mean $\left[\mathrm{Ca}^{2+}\right](\mathrm{nM}) \pm$ s.e.m. (one-way ANOVA; $n=3$ ). i. Transcript abundance of $P M C A$ compared to $R p L 32$ expression in muscles from control (+/), knockout $\left(h o w^{t s}>\operatorname{CapaR}^{K O}\right)$ and rescue $\left(h o w^{t s}>\operatorname{CapaR}^{K O}\right.$, CapaR) flies (one-way ANOVA; n=5). 
a

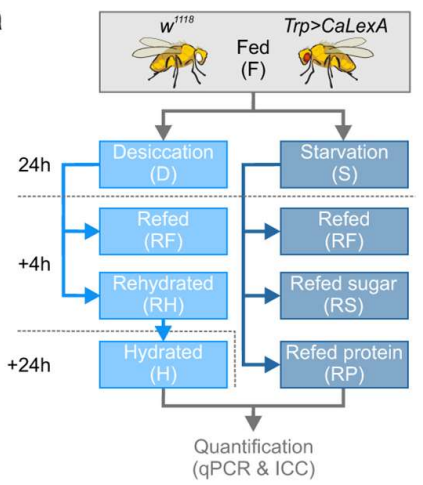

c

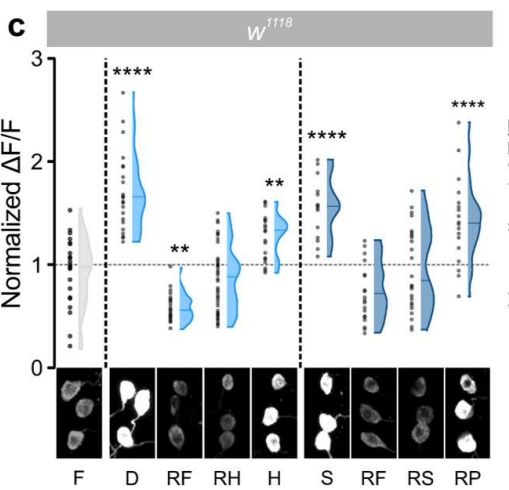

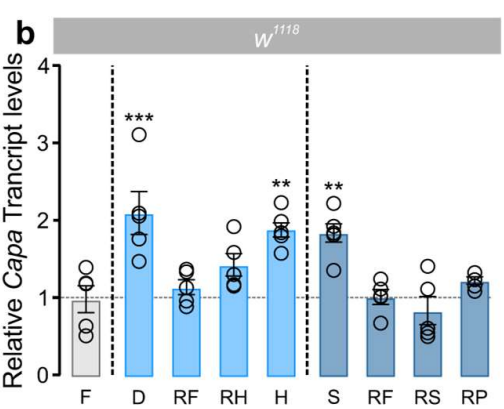

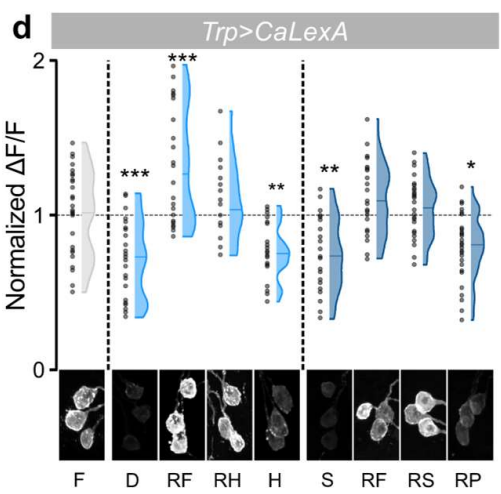

Figure 7

Figure 7. Environmental cues modulating $\mathrm{Capa}^{+}$Va neuron activity. a. Experimental design for the environmental exposures of wild-type $\left(w^{1118}\right)$ and $\operatorname{Tr} p>C a L e x A$ flies. b. Transcript levels of Capa gene expression relative to $R p L 3$ in Va neurons (thorax samples) of flies exposed to the environmental conditions indicated (one-way ANOVA; $n=5$ ). c. Violin plots and raw data points of immunofluorescence quantifications of intracellular Capa precursor levels ( $\mathrm{n}=18-52)$ and d. CaLexA-induced GFP expression $(\mathrm{n}=18-36)$ in $\mathrm{Capa}^{+} \mathrm{Va}$ neurons with representative images from all conditions (one-way ANOVA). 

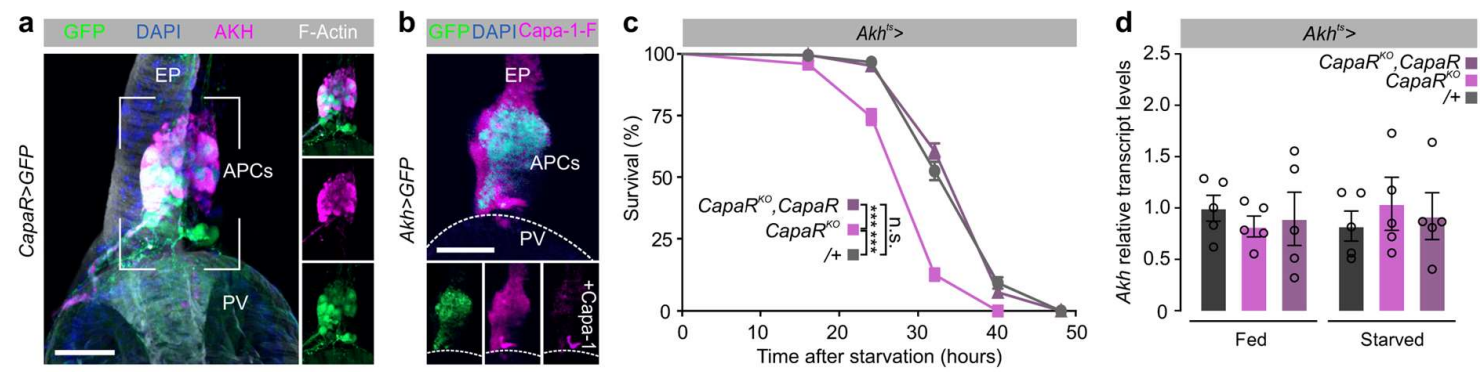

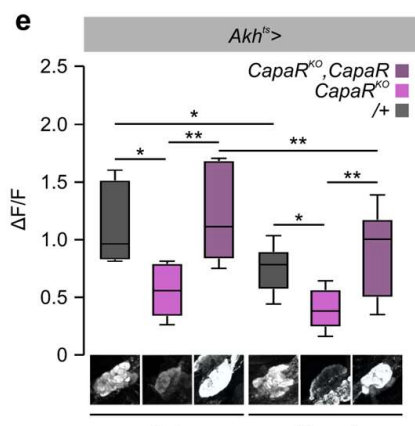

Fed

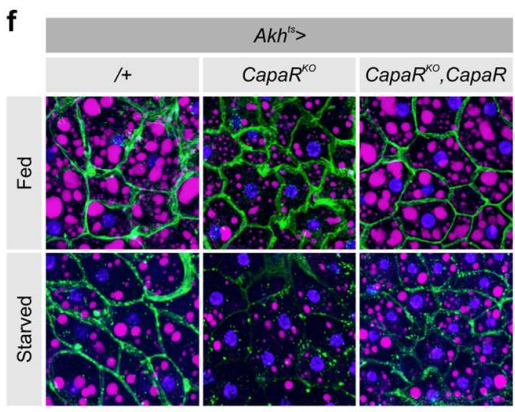

F-Aktin DAPI NileRed
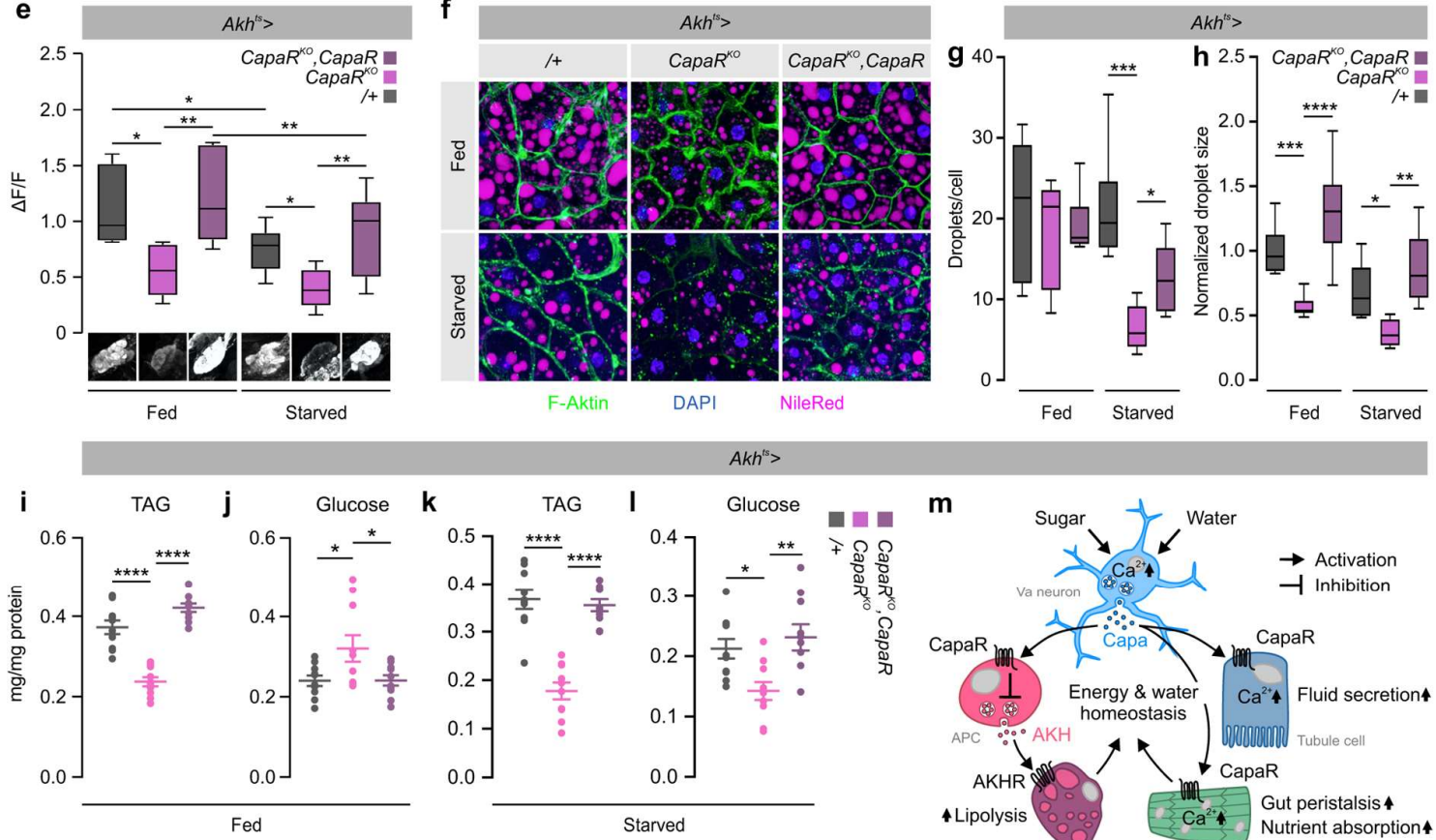

Figure 8

Figure 8. Systemic Capa signaling regulates metabolic homeostasis via AKH activity. a.

AKH immunofluorescence (magenta) colocalizes with CapaR $>$ driven $m C D 8:: G F P$

expression (green) confirming CapaR localization to the APCs. Scale bar $10 \mu \mathrm{m}$. b.

Application of Capa1-F (magenta) shows specific and displaceable binding to $A k h>G F P$

(green) positive cells in the APCs. Scale bar $10 \mu \mathrm{m}$. EP, esophagus; PV, proventriculus;

APCs, AKH-producing neurosecretory cells. c. CapaR knockout in the APCs

$\left(A k h>\operatorname{CapaR}^{K O} ; \mathrm{n}=208\right.$, ) results in significantly reduced resistance to starvation compared to parental controls (+/; n=213), while co-overexpression of CapaR $\left(\right.$ Akh $>$ CapaR ${ }^{K O} ;$ CapaR;

$\mathrm{n}=247$,) rescues the survival phenotype (log-rank test). d. Transcript levels of $A \mathrm{kh}$ relative to

RpL32 in the APCs from the different genetic backgrounds (one-way ANOVA; $\mathrm{n}=5$ ). e. 
Immunofluorescence measurements of intracellular AKH levels in the APCs from animals exposed to either ad libitum feeding (fed) or caloric deprivation (starved) for 24 hours, probed with an anti-AKH antibody (one-way ANOVA; $n=6-15)$. f. Lipophilic dye Nile red staining of adult dissected fat bodies from fed and starved control (/+), knockout (CapaR $\left.{ }^{K O}\right)$ and rescue flies $\left(\operatorname{CapaR}^{K O}\right.$, CapaR). g. Quantification of lipid droplets/cell and h. lipid droplet size in fed and starved flies with the different genetic backgrounds (one-way ANOVA; $n=6-8$ ). i.-l. Quantification of whole-body TAG and $\mathbf{j}$. glucose in fed and starved animals of the different genotypes (one-way ANOVA; $n=10$ ). m. Working model for the systemic actions of Capa/CapaR signaling in mediating water and osmotic homeostasis in adult Drosophila. $\mathrm{Capa}^{+}$Va neurons respond to internal sugar and osmotic cues by activating two separate pathways: one stimulating intestinal and renal activities to promote gut contractions, nutrient absorption and fluid secretion, and another to restrict glucagon-like AKH release from the APCs and subsequent lipolytic activity in adipose tissue. 


\section{SUPPLEMENTAL INFORMATION}

a
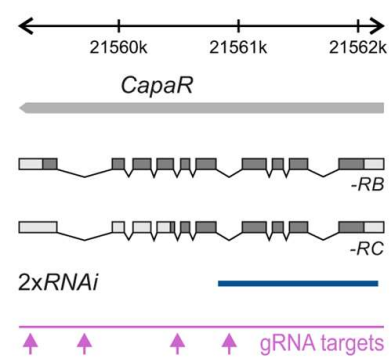

b

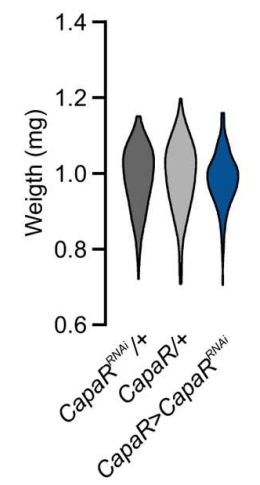

e

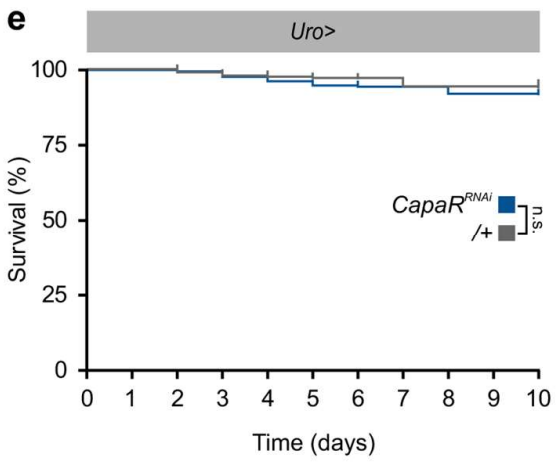

i

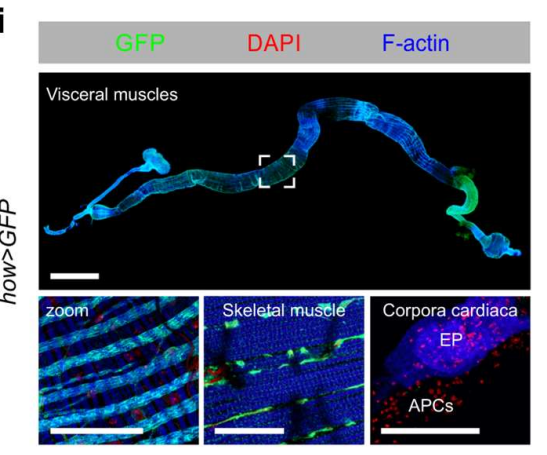

C
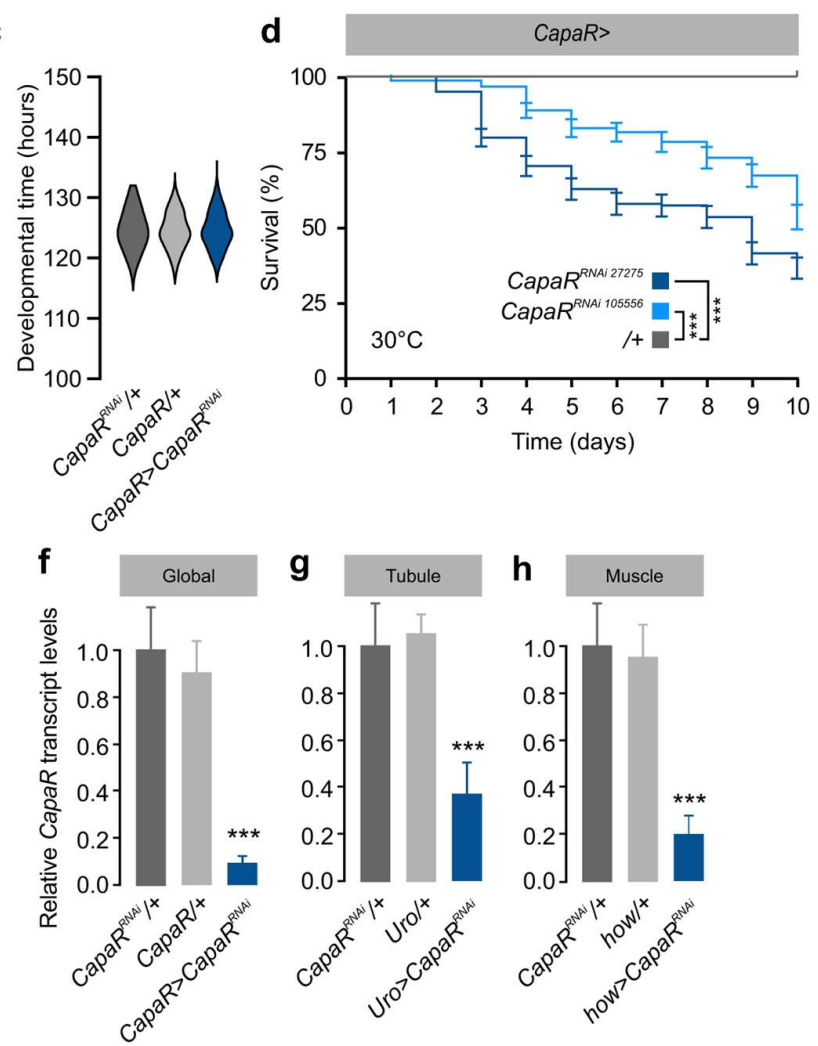

h Muscle
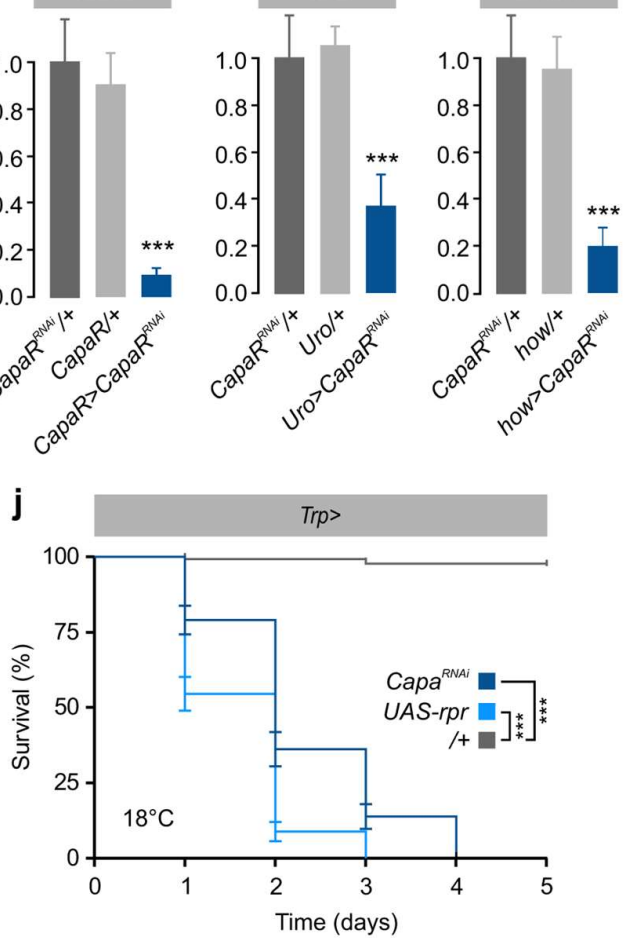

Figure S1. Extra-renal Capa/CapaR signaling is essential for adult fly viability. a. Exon map of the CapaR gene indicating the regions targeted by RNAi (blue; in-house generated RNAi line) and CRISPR/Cas9-mediated (magenta) genome editing techniques. b. Pupal weight of global CapaR knockdown flies (CapaR>CapaR $\left.{ }^{R N A i} ; \mathrm{n}=134\right)$ is not significantly different compared to $\operatorname{CapaR}>(\mathrm{n}=111)$ and $\operatorname{CapaR}^{R N A i} /+(\mathrm{n}=140)$ parental controls (one-way ANOVA). c. Developmental time of global CapaR knockdown flies (CapaR $>$ CapaR ${ }^{R N A i}$; 
$\mathrm{n}=129)$ is not significantly different compared to $\operatorname{CapaR}(\mathrm{n}=107)$ and $\mathrm{CapaR}^{R N A i} /+(\mathrm{n}=123)$ parental controls (one-way ANOVA). d. Two independent CapaR $^{R N A i}$ lines, CapaR ${ }^{R N A i} 27276$ $(\mathrm{n}=183)$ and $\operatorname{CapaR}^{R N A i} 105556(\mathrm{n}=151)$, show the same mortality phenotype relative to control $(n=151)$ confirming the specificity of the RNAi-effect (log-rank test). e. Knockdown of CapaR in tubule principal cells $(\mathrm{n}=287)$ did not induce significant changes in fly survival compared to control (log-rank test; $\mathrm{n}=231$ ). f.-h. CapaR transcript abundance normalized to RpL32 following RNAi-mediated CapaR knockdown d. globally, e. in Malpighian tubules, and f. in muscles (one-way ANOVA; $\mathrm{n}=5$ ). i. Analyzing how $>m C D 8:: G F P$ (green) confirm GAL4-driven expression in visceral and skeletal muscles, but not the $\mathrm{CC}$, of adult Drosophila. Scale bar $75 \mu \mathrm{m}$. j. Knocking down Capa expression $(\mathrm{n}=72)$ or completely ablating $(\mathrm{n}=70)$ the Capa-producing neurons by ectopic expression of $r p r$, phenocopies the mortality of CapaR silencing in adult Drosophila relative to control (log-rank test; $\mathrm{n}=133$ ). 
a

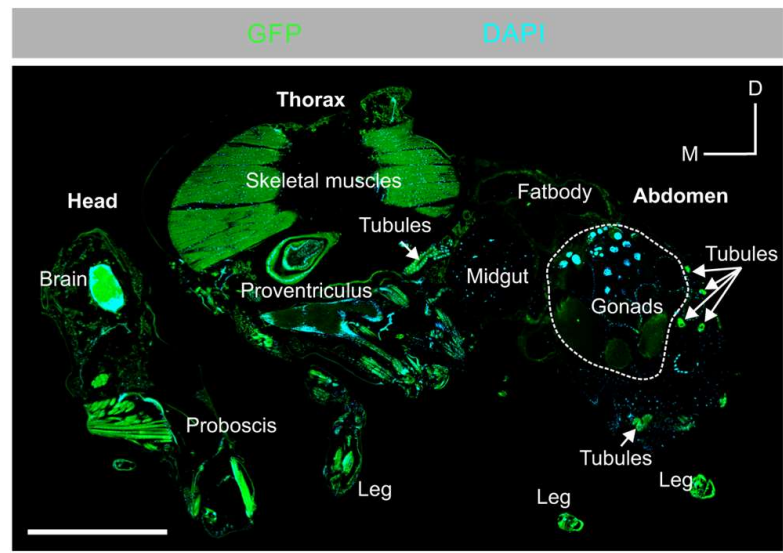

d

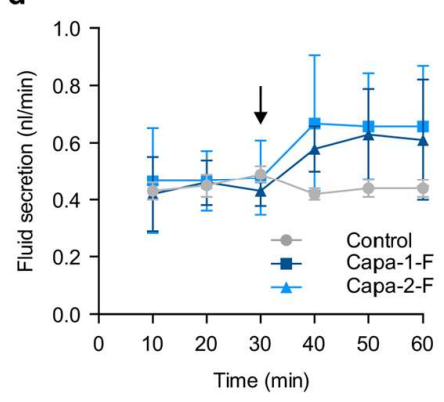

f
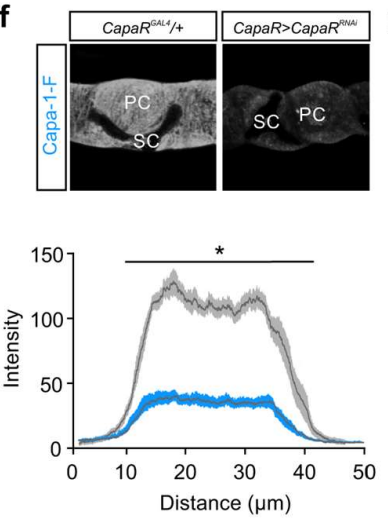

e

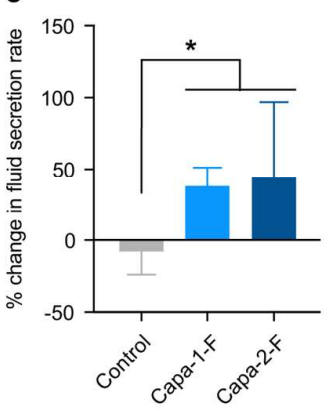

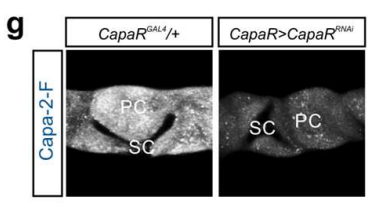

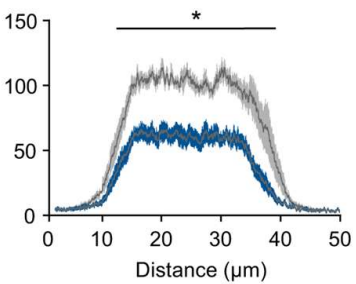

b

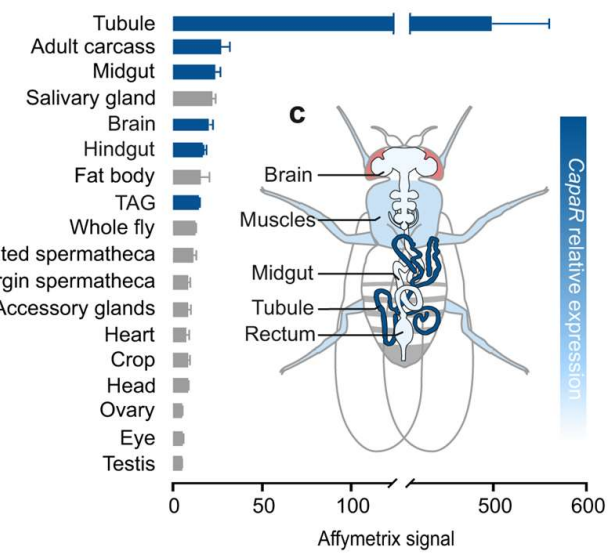

h

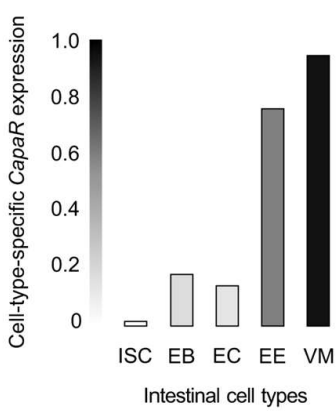

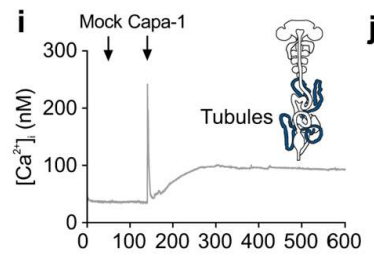
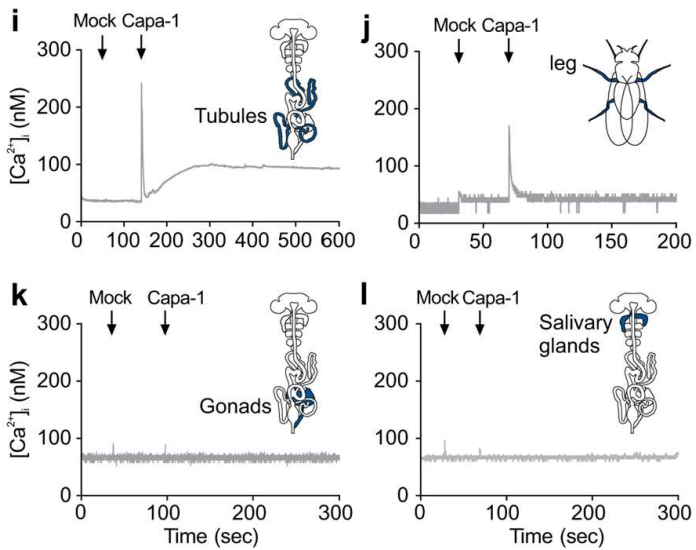

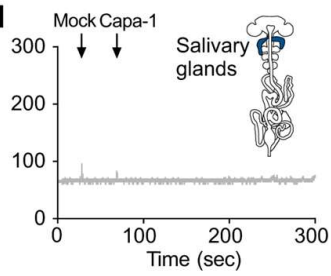

Figure S2. Spatial expression analysis and functional validation of CapaR. a. Medial paraffin section of adult fly carrying CapaR> and UAS-mCD8::GFP showing GFP expression in different tissues, including the tubules, brain, somatic musculature, and gut. $\mathbf{b}$. Mean normalized Affymetrix signal \pm s.e.m showing CapaR spatial expression across major adult tissues. c. Relative CapaR expression superimposed on an anatomical map of adult Drosophila. d.-e. Validation of fluorophore-coupled Capa-1 (Capa-1-F) and Capa-2 (Capa-2F) peptides. d. Fluid secretion assays show significant functional stimulation of renal tissues 
by Capa-1-F and Capa-2-F peptides demonstrating biological activity. e. Percent change in fluid secretion rate following addition of Capa-1-F or Capa-2-F ligands (one-way ANOVA; $\mathrm{n}=7$ ). f. Knockdown of CapaR expression significantly reduces fluorescent intensity in principal cells compared to parental controls following Capa-1-F and Capa-2-F application (one-way ANOVA; $\mathrm{n}=5$ ). $\mathbf{h}$. Relative expression of CapaR in the different cell types of the intestine as realized by FACS sorted cell populations. Intestinal stem cells, ISC; Enteroblasts, EB; Enterocytes, EC; Enteroendocrine cells, EEs; Visceral muscles, VM. Data from: flygutseq.buchonlab.com ${ }^{62}$. i.-l. Functional validation of tissue-specific Capa signaling using in vivo calcium-reporter technology. Stereotypic biphasic increases in cytosolic $\left[\mathrm{Ca}^{2+}\right]_{\mathrm{i}}$ was detected upon Capa-1 application $\left(10^{-7} \mathrm{M}\right)$ in i. tubules, and $\mathbf{j}$. legs, but not in $\mathbf{k}$. testes or $\mathbf{l}$. salivary glands. 


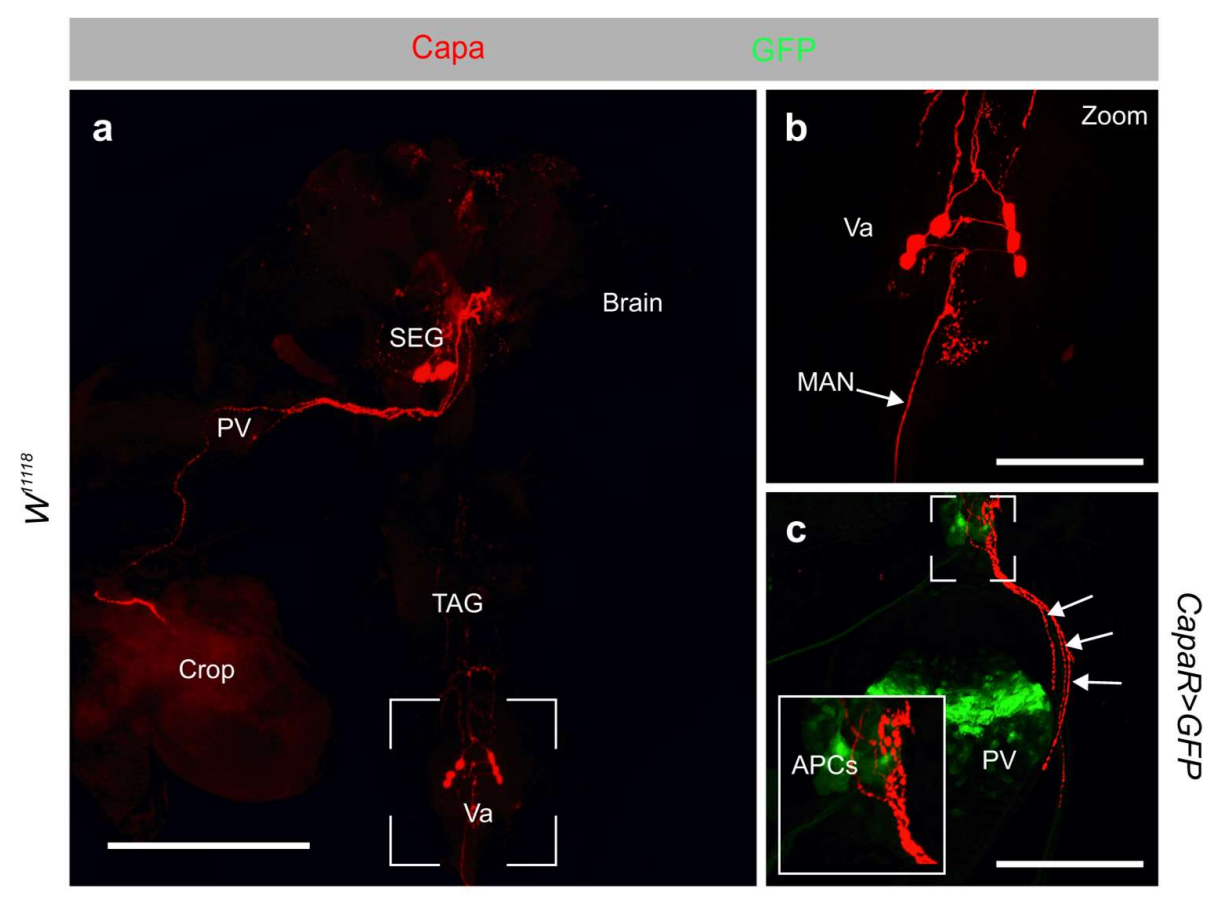

Figure S3. Neuroanatomy of Capa ${ }^{+}$neurons. a. Immunoreactivity of Capa precursor antibody (red) identifies one pair of SEG neurons and three pairs of $\mathrm{Va} \mathrm{Capa}^{+}$neurons. Scale bar $=200 \mu \mathrm{m}$. b. The Va neurons release Capa peptides into circulation via the median abdominal neuron (MAN). Scale bar $=100 \mu \mathrm{m}$. c. The axons from the SEG neurons innervate the AKH-producing neuroendocrine cells (APCs) and proventriculus (PV), which also shows CapaR $>m C D 8:: G F P$ expression (green) in muscle cells. Scale bar $=100 \mu \mathrm{m}$. 


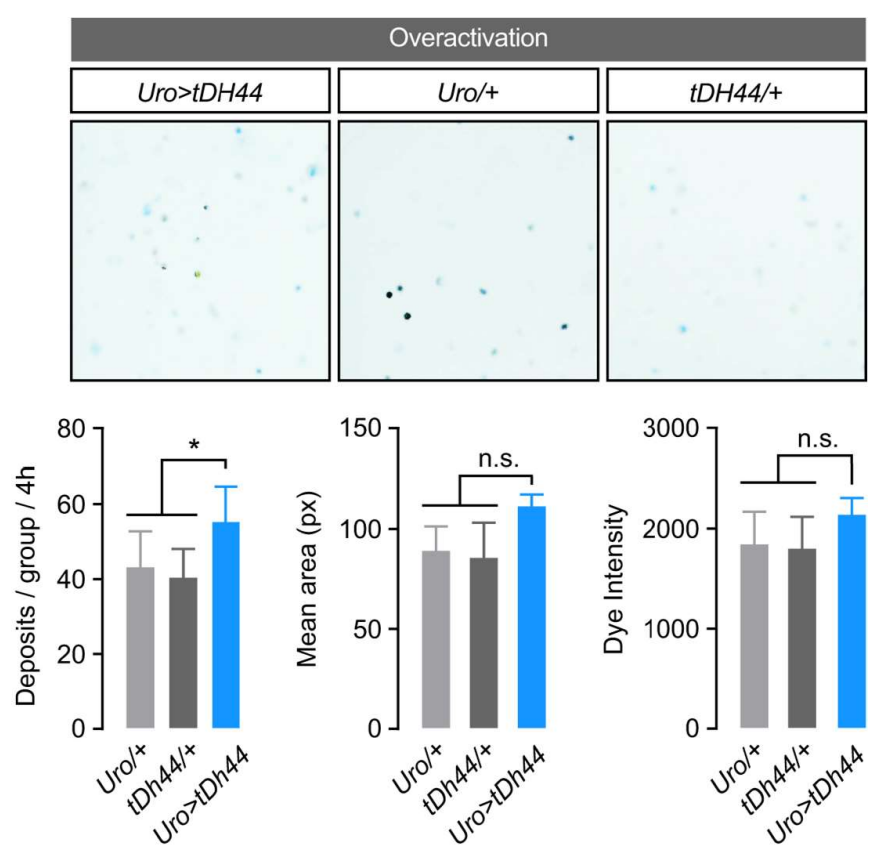

Figure S4. Functional stimulation of renal tubules increase waste excretion. Fecal output profiles following artificial activation of renal secretion by ectopic expression of a membrane-tethered version of diuretic hormone 44 (tDH44) show that these flies only produce more, but not larger of more dilute, deposits compared to control (one-way ANOVA; $n=5-7)$. 

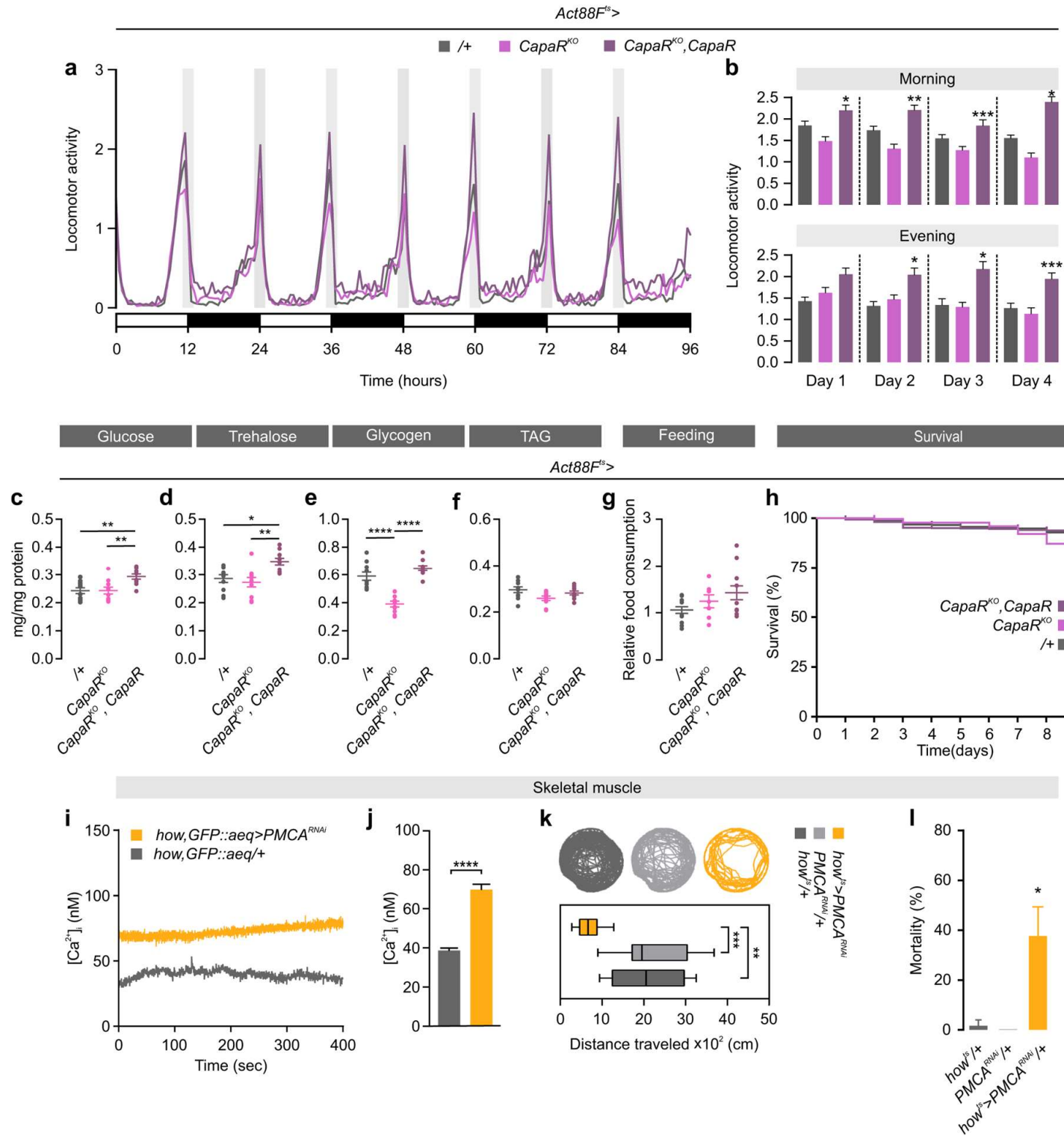

Figure S5. Physiological effects of eliminating Capa signaling in skeletal muscles. a.

Locomotor activity of individual control $(+/ ; n=32)$, skeletal muscle-specific CapaR

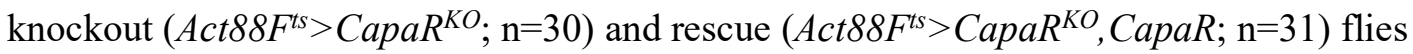

exposed to 12-hour:12-hour, light-dark (LD) cycles over 96 hours. b. Morning and evening activity peaks measured during the experimental period (one-way ANOVA). c.-f.

Quantification of c. glucose, d. trehalose, e. glycogen and f. TAG levels in flies with the different genotypes (one-way ANOVA; $n=10$ ). g. Relative food intake (one-way ANOVA $\mathrm{n}=10)$. h. Kaplan-Meier survival curves of control $(+/ ; \mathrm{n}=508)$, knockout 


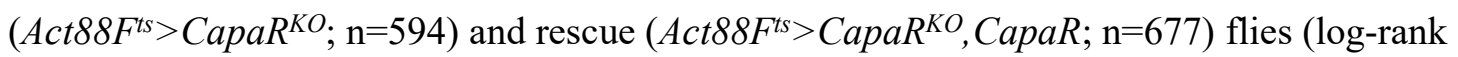
test). i.-j. Quantification of $\left[\mathrm{Ca}^{2+}\right]_{\text {i }}$ following $P M C A$ knockdown in skeletal muscles (how, GFP::aeq $>P M C A^{R N A i}$ ) relative to control (how, GFP::aeq/+; Student's $t$-test; $\mathrm{n}=3$ ). k. Representative activity traces of video-tracked individual flies with targeted PMCA knockdown in muscles $\left(h o w^{t s}>P M C A^{R N A i} ; \mathrm{n}=10-16\right)$ relative to control flies, including quantification of distance travelled (one-way ANOVA). 1. Knockdown of $P M C A$ in muscles $\left(h o w^{t s}>P M C A^{R N A i}\right.$ ) causes significant fly mortality (one-way-ANOVA; $\mathrm{n}=60$ ). 

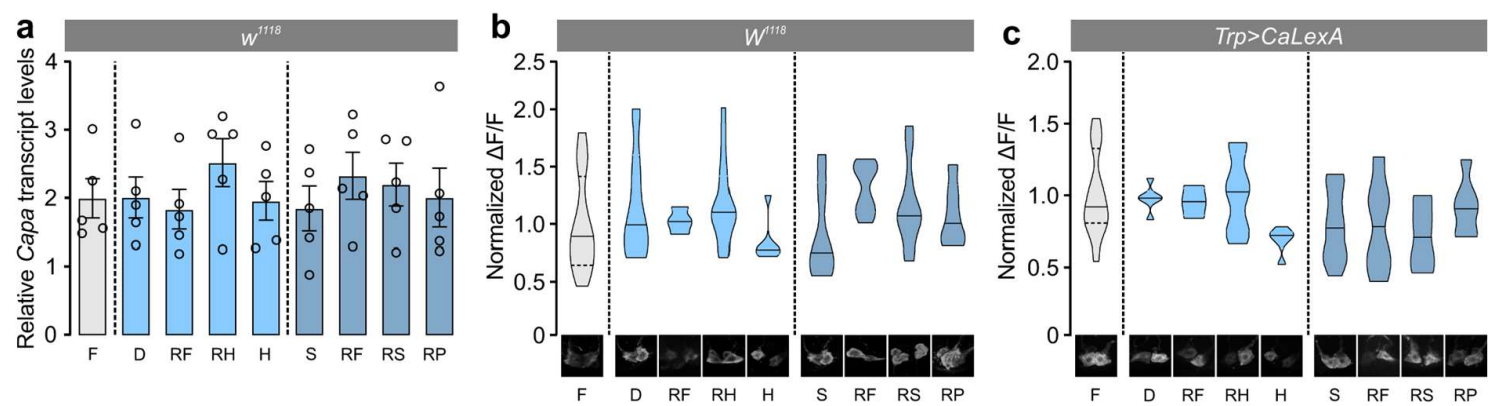

Figure S6. Capa $^{+}$SEG neuron activity is unaffected by water or nutrient stress. a.

Transcript levels of Capa relative to $R p L 3$ in the SEG neurons (head samples) from flies exposed to different environmental conditions (one-way ANOVA; $\mathrm{n}=5$ ). b. Violin plots of immunofluorescence quantifications of intracellular Capa precursor levels $(n=6-16)$ and $\mathbf{c}$. CaLexA induced GFP expression in Capa-producing SEG neurons $(\mathrm{n}=4-12)$ from flies exposed to different environmental conditions (one-way ANOVA). F, fed; D, desiccated; RF, refed; RH, rehydrated; H, hydrated; S, starved; RS, refed sugar; RP, refed protein. 
Table S1. Primer sequences used in Drosophila transgenesis.

\begin{tabular}{|l|l|}
\hline & Primer sequence \\
\hline NotI-CapaR_F & 5'-CGCGGCCGCATGAATTCATCGACCGATCCGAC-3' \\
\hline KpnI-CapaR_R & 5'-GCGGTACCTTAAATACAAGTCTCCTCGTTCTCGATCC-3' \\
\hline CapaR_CRISPRI_F & 5'-CGGCCCGGGTTCGATTCCCGGCCGATGCAGCTGTTGGATGGTGAAG \\
\hline CapaR_CRISPRI_R & 5'-AATCAATCCCAACAGAATCCTGCACCAGCCGGGAATCGAACC-3' \\
\hline CapaR_CRISPR2_F & 5'-GGATTCTGTTGGGATTGATTGTTTCAGAGCTATGCTGGAAAC-3' \\
\hline CapaR_CRISPR2_R & 5'-GCTTGTATTCCACGTGTGTATGCACCAGCCGGGAATCGAACC-3' \\
\hline CapaR_CRISPR3_F & 5'-TACACACGTGGAATACAAGCGTTTCAGAGCTATGCTGGAAAC-3' \\
\hline CapaR_CRISPR3_R & 5'-ATTTTAACTTGCTATTTCTAGCTCTAAAACAGTCATATCACTAAATT \\
& CCCTGCACCAGCCGGGAATCGAACC-3' \\
\hline
\end{tabular}

Table S2. Quantitative RT-PCR primers used.

\begin{tabular}{|c|c|}
\hline Primer name & Primer sequence \\
\hline CapaR_F & 5'-TACCCCAGATACCCTATTCGCC-3' \\
\hline CapaR_R & 5'-CTAAGAGATTACCAACGACCCCG-3' \\
\hline GlyS_F & 5'-GCAAGCTGTTTACCCAGACTATGTGG-3' \\
\hline GlyS_R & 5'-CAATTCCTTGAGTTCGGTCTCCTC-3' \\
\hline$A G B E \_F$ & 5'-CGGCGATTACAACGAGTACTTTGG-3' \\
\hline$A G B E \_R$ & 5'-GGTAGGCATTCCAGAAACATCCTC-3' \\
\hline GlyP_F & 5'-AGTACGACCACTACTACTTGCTGG-3' \\
\hline$G l y P_{-} R$ & 5'-CGCTGGACGCAATGTTGTTGATGG-3' \\
\hline$A G L_{-} F$ & 5'-GATTCAGTCCTGCTCTAAGTCCAC-3' \\
\hline$A G L \_R$ & 5'-CACGCAGAGCGATGAAGGTATCAC-3' \\
\hline$A k h_{-} F$ & 5'-TCCCAAGAGCGAAGTCCTCA-3' \\
\hline
\end{tabular}


bioRxiv preprint doi: https://doi.org/10.1101/2020.07.24.219592; this version posted July 25, 2020. The copyright holder for this preprint (which

was not certified by peer review) is the author/funder, who has granted bioRxiv a license to display the preprint in perpetuity. It is made available under aCC-BY-NC-ND 4.0 International license.

\begin{tabular}{|l|l|}
\hline$A k h_{-} R$ & 5'-GTCCAGAAAGAGCTGTGCCT-3' \\
\hline$P M C A \_F$ & 5'-TCTATTGCTCCGCAAACCCTATGG-3' \\
\hline$P M C A \_R$ & 5'-GTCCACGTCCAGATTCAATATCGAG-3 \\
\hline$R p L 32_{-} F$ & 5'-TGACCATCCGCCCAGCATAC-3' \\
\hline$R p L 32_{-} R$ & 5'-ATCTCGCCGCAGTAAACG-3' \\
\hline$R p L 3 \_F$ & 5'-AAGGATGACGCCAGCAAGCCAGTC-3' \\
\hline$R p L 3 \_R$ & 5'-TAGCCGACAGCACCGACCACAATC-3' \\
\hline
\end{tabular}

